\title{
Peuplement et trajectoires dans l'espace résidentiel : le cas de la
} Seine-Saint-Denis

J.-P. Lévy

\section{Citer ce document / Cite this document :}

Lévy J.-P. Peuplement et trajectoires dans l'espace résidentiel : le cas de la Seine-Saint-Denis. In: Population, $58^{\mathrm{e}}$ année, $\mathrm{n}^{\circ} 3$, 2003. pp. 365-400;

http://www.persee.fr/doc/pop_0032-4663_2003_num_58_3_7399

Document généré le 11/06/2016 


\title{
Résumé
}

Levy Jean-Pierre.- Peuplement et trajectoires dans l'espace résidentiel : le cas de la Seine- SaintDenis L'article porte sur les processus de transformation du peuplement du département de la SeineSaint-Denis (banlieue nord et est de Paris, 500000 ménages), du triple point de vue des logiques résidentielles des groupes sociaux, des effets de la structure de l'offre et des marquages sociaux des territoires. À partir des données du recensement général de la population de 1990, on regroupe en classes d'habitat des types de logements dont les habitants ont des caractéristiques proches. Ces classes sont ensuite hiérarchisées et interprétées à partir d'un indice de statut socio- résidentiel, dont les variations spatiales nous permettent d'identifier l'influence des marquages sociaux des communes sur le peuplement (aires résidentielles), qu'ils relèvent des composantes de leur parc immobilier (zones d'habitat) ou de leur situation géographique (effets locaux). Ces typologies sont ensuite utilisées pour interpréter la mobilité résidentielle des ménages entrés dans un logement de la Seine-Saint-Denis en 1994. Les données relatives aux 1568 ménages enquêtes ont été exploitées dans une triple direction : pour cerner les trajectoires résidentielles des ménages; pour mettre à jour les choix de localisation des ménages, les marges de manœuvre et les contraintes pesant sur leur décision; en utilisant les parcours résidentiels du moment comme un indicateur des évolutions des peuplements territoriaux.

\begin{abstract}
Lévy Jean-Pierre.- Population Patterns and Household Trajectories in the Residential Milieu: the Example of the Seine-Saint-Denis This article examines the processes underlying changes in the residential population of the Seine-Saint-Denis department (a suburban district to the north and east of Paris, containing 500,000 households) from three points of view: 1) the residential logics of different social groups, 2) the effects of the housing supply structure and 3) of the social markers attributed to the different local areas. Data from the 1990 Census are used to establish housing categories containing housing types whose residents have similar characteristics. These categories are then ranked and interpreted using an index of socio-residential status, whose spatial variations indicate the influence of the social markers of the communes on the population (residential areas), which can derive from the composition of their housing stock (housing zones) or from their geographical situation (local effects). These typologies are then used to interpret the residential mobility of households who moved into a dwelling in the Seine-Saint-Denis in 1994. Data pertaining to the 1,568 surveyed households are analysed in three ways: to determine the residential trajectories of households; to shed light on the locational choices of households, and on the scope for action and constraints shaping their decision; and current residential histories are used as an indicator of changes in local populations.
\end{abstract}

\section{Resumen}

Levy Jean-Pierre.- Población y trayectorias residenciales: el ejemplo de Sena- San Denis Este artículo analiza el proceso de transformación del poblamiento del departamento de Sena- San Denis (distrito del area metropolitana, situado al noreste de Paris, 500.000 ho- gares) desde très perspectivas: las estrategias residenciales de diferentes grupos sociales, el impacto de la estructura de la oferta y las demarcaciones o estructura social del territorio. A partir de los datos del censo general de población de 1990 se construyen categorías de habitat en base a tipos de vivienda cuyos résidentes tienen caracteristicas similares. Estas categorías son ordenadas jerárquicamente y se interpretan en base a un indice de status socio- residencial, las variaciones espaciales del cual permiten estudiar la influencia de la categoria social del municipio sobre su poblamiento (areas residenciales), tanto en lo relativo al parque inmobiliario (zonas de vivienda) como a su situación geográfica (efectos de localización). Estas tipologías sirven de base para estudiar la movilidad residencial de los hogares que se instalaron en Sena - San Denis en 1994. La explotación de los datos relativos a los 1,568 hogares encuestados tiene très objetivos: observar las trayectorias residenciales de los hogares, describir la selección de localización de los hogares, las opciones disponibles y las restricciones existentes a la hora de tomar una decision. Las trayectorias residenciales se uti- lizan como indicador de la evolución del poblamiento territorial. 


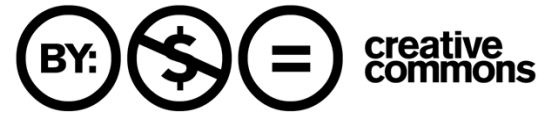




\title{
Peuplement et trajectoires dans l'espace résidentiel : le cas de la Seine-Saint-Denis
}

\author{
Jean-Pierre LÉVY*
}

\begin{abstract}
La prise en compte de plusieurs niveaux d'analyse afin de mieux comprendre les comportements constitue un des nouleatux défis de la recherche en sciences humaines. Pour cerner les facteurs d'évolution du peuplement des communes ainsi que les stratégies des ménages qui s'y installent, y restent ou les quittent, Jean-Pierre Lévy propose une démarche qui associe les caractéristiques physiques de l'habitat aux positions sociales des habitants. Il prend ensuite en compte le fait que le statut de tel ou tel trpe de logement relève au moins autant du voisinage, du quartier, des équipements, én un mot de son environnement. La méthode. mise en xuvre pour traiter de l'évolution du peuplement des communes d'un département de la banlieue parisienne, permet également de décrire les trajectoires dé ses habitants.
\end{abstract}

Le rôle de l'habitat dans les formes de peuplement territoriales n'est . pas à proprement parler une question nouvelle posée aux chercheurs sur la ville. La géographie régionaliste du début du XXe siècle - à dominante ruraliste, il est vrai - (Demangeon, 1921), les travaux sur la ségrégation sociale ou démographique (Brun et Rhein, 1994 ; Le Bras et Chesnais, 1976), voire la littérature économique sur les valeurs foncières et immobilières (Gaubert et al., 1996: Calcoen et Cornuel, 1999) ont déjà largement abordé cette question. Dans le contexte contemporain, elle tend cependant à se complexifier du fait notamment de nouvelles formes de territorialisation (Berger et Rhein, 1988; Arbonville et Bonvalet, 1992), d'associations moins mécaniques entre les caractéristiques du parc de logements et celles de leurs occupants (Ballain et al., 1984 ; Lévy 1995. 1998a et (998b) et de la prise en compte plus systématique des marges de

* CNRS/CRH-Louest (UMR 7145 du CNRS).

Cet article est issu d une étude réalisée au Centre de recherche sur l'habitat (C'RH) pour le compte de la Direction départementale de l'équipement (DIDF) el du Conséil général de SeinéSaint-Denis (cf. Lévy et Haumont. 1997). 
manouvre des habitants dans l'étude des choix résidenticls (Clark et Onaka, 1983; Courgeau, 1985 ; Lelièvre, 1992 ; Clark et Dielman, 1996 ; Bonvalet, 1997 ; Baccaïni, 2000). Si chacun de ces éléments a pu être étudié dans différents contextes et à différentes échelles, les articulations entre ces facteurs et leurs effets sur le peuplement restent à explorer. Dans un contexte urbain donné, comment les ménages arbitrent-ils entre localisation et type d'habitat? De quelles marges de manceuvre disposent-ils pour ce faire? Dans quelle mesure ces arbitrages influencent-ils les évolutions du peuplement local et l'organisation des parcours résidentiels dans la société globale?

Cet article propose une méthode d'analyse susceptible de répondre à ces questions, grâce à l'étude de l'organisation socio-territoriale d'un parc immobilier et de la mobilité résidentielle qui lui est associéc. Cette méthode est utilisée pour décrire les dynamiques de peuplement à l'ceuvre au milieu des années 1990 dans un département de la banlieue nord et est de Paris, la Seine-Saint-Denis, qui compte 500000 ménages. Son parc de logements se caractérise par la présence d'un habitat ancien, renouvelé au cours du siècle par la construction de maisons individuelles en lotissements, généralement périurbains, et des logements sociaux construits massivement dans les années 1960 et 1970. La crise industrielle des années 1970 a bouleversé l'espace social et les proportions élevées de jeunes n'ayant jamais travaillé, de chômeurs de longue durée, de familles monoparentales et de ménages aux faibles ressources sont aujourd hui autant de caractéristiques qui pèsent sur la structure sociodémographique de la population du département. Néanmoins, malgré l'importance de la population fragilisée, la Seine-Saint-Denis attire et stabilise une fraction de ménages mieux armés du point de vue socio-économique. Ils appartiennent aux couches moyennes et supérieures de la société : il peut s'agir de jeunes ménages en provenance d'autres agglomérations ayant trouvé un emploi qualifié dans ce département en crise. de jeunes couples attirés par les faibles valeurs foncières, mais également de familles durablement installées dans ces espaces résidentiels. Ces groupes participent à un niveau ou un autre à l'embourgeoisement de secteurs particuliers du département. Le choix de ce site offre donc l'opportunité d'une comparaison exemplaire des dualités entre les populations sous contrainte et les autres. Par ailleurs, les diversités sociales internes au département permettent de comparer les communes en insistant sur les effets des marquages sociaux des territoires.

À travers l'étude de la mobilité, nous cherchons à comprendre dans quelle mesure et comment les logiques résidentielles des habitants influencent le peuplement des territoires urbains et son évolution. Notre objectif est cependant de dépasser les questions d'ordre méthodologique, pour appréhender plus largement les diversités et les récurrences dans l'organisation spatiale de l'habitat et dans le peuplement des espaces résidentiels français. C'est la raison pour laquelle, dans un premier temps, nous serons amené à privilégier l'étude des interactions entre deux dimensions, à 
savoir l'offre immobilière et ses effets sur l'attraction ou la répulsion des différentes catégories de ménages dans l'espace urbain, d’une part, et les marquages sociaux des territoires susceptibles d'influer sur les formes de peuplement en place ou à venir, d'autre part. La connaissance de ces interactions nous permettra dans un second temps d'étudier de façon originale l'influence conjointe des facteurs sociaux et démographiques sur les mobilités résidentielles et, surtout, de mettre en évidence l'adaptation de ces mouvements aux conjonctures locales.

\section{Structures de l'habitat et du peuplement}

\section{Occupation des logements : classes d'habitat et statuts socio-résidentiels}

Il est difficile de comparer les structures d'occupation de différents sites en se référant aux seules caractéristiques morphologiques du bâti des immeubles d'habitation. Dans un milieu social donné, le statut que confère à son occupant tel ou tel type d'habitat relève tout autant des caractéristiques du logement et de l'immeuble que de son environnement au sens large (par exemple le voisinage, la composition sociale de la population du quartier ou son équipement). Des travaux antéricurs ont d'ailleurs déjà montré l'adaptation des choix résidentiels des habitants aux contextes immobiliers locaux (Lévy, 1995). Il en découle qu'unc comparaison des structures d'occupation basée sur les seules caractéristiques des logements a toutes les chances d'aboutir au constat d'une hétérogénéité des fonctions sociales de chacun des types d'habitat.

Pour mener à bien une étude des dynamiques du peuplement du triple point de vue des logiques résidentielles des individus ou des groupes sociaux. des effets de la structure de l'offre et des marquages sociaux des territoires, il est donc nécessaire d'élaborer une méthode qui puisse être mise en ouvre quelles que soient les caractéristiques du bâti et des sites. Cette méthode doit notamment éliminer deux biais importants : d'une part, ceux introduits par la présence d'un type d'habitat particulier dans un site donné (les types d'habitat ne pouvant alors être comparés avec profit): d'autre part, ceux dus aux variations des caractéristiques des occupants d un type de logement selon les sites (qui amènent généralement à assimiler des types d'habitat n'ayant pas le même statut social dans chacun des lieux).

Pour s'affranchir de ces difficultés. nous proposons une nouvelle classification de l'habitat basée non pas sur les aspects morphologiques ou juridiques du bâti, mais sur les caractéristiques des habitants. Pour cela. nous avons construit une variable synthétique. le statut socio-résidentiel des types de logements, qui illustre les liens symboliques associant les caractéristiques du logement à la position sociale de son occupant. Ce statut 
est déterminé en s'appuyant sur le rapport entre les caractéristiques morphologiques du parc immobilier et celles de ses habitants.

La méthode consiste à regrouper en classes d'habitat des types de logements dont les habitants ont des caractéristiques identiques. Ces classes sont ensuite hiérarchisées et interprétées à partir d'un indice de statut socio-résidentiel. Les variations spatiales des valeurs de cet indice nous permettent alors d'identifier l'influence des marquages sociaux des communes sur le peuplement (aires résidentielles), qu'ils relèvent des composantes de leur parc immobilier (zones d'habitat) ou de leur situation géographique (effets locaux).

La première étape consiste donc à élaborer une grille d'analyse qui permette de comparer et de classer tous les logements quel que soit le site étudié. Cette grille doit être la même pour les ménages sédentaires et pour la population mobile, dont on veut précisément tester la propension à se diriger vers tel ou tel habitat. Grâce à un traitement secondaire des données du recensement au quart de la population française réalisé en 1990, nous construisons dans un premier temps une liste des types de logements potentiellement présents dans le département en croisant deux catégories de variables: les premières concernent le type d'immeuble et son statut juridique (habitat individuel ou collectif, date de construction, parc privé ou parc social); les secondes sont relatives au logement (statut d'occupation, confort, nombre de pièces).

Au total, nous identifions ainsi 144 types théoriques de logement possibles. Mais pour isoler un type, nous nous sommes fixé un seuil minimum (égal à 1000 logements présents dans la base non pondérée). Des regroupements sont donc effectués à partir d'une arborescence, lesquels aboutissent à ne conserver que 31 types de logements (voir annexe).

Dans un deuxième temps, nous déterminons pour chacun de ces 31 types de logements les caractéristiques socio-économiques des ménages qui les occupent : la catégorie socioprofessionnelle de la personne de référence, sa nationalité (Français ou étranger), son statut d'activité (actif occupé ou chômeur) (tableau 1 ).

Dans un troisième temps, à partir d'une analyse en composantes principales (ACP), nous établissons les correspondances entre les types de logements et les caractéristiques socioprofessionnelles des ménages occupants (figure l); enfin, à l'aide d'une classification hiérarchique ascendante, nous regroupons en trois classes les types de logements dont les occupants ont des caractéristiques proches.

Chacune des classes regroupe environ un tiers des logements du département. La première classe réunit 29000 logements $(31 \%$ du parc immobilier total); la deuxième comprend un peu plus de 30000 logements (33\% du total) et la troisième, plus de 33000 logements (36\%). 


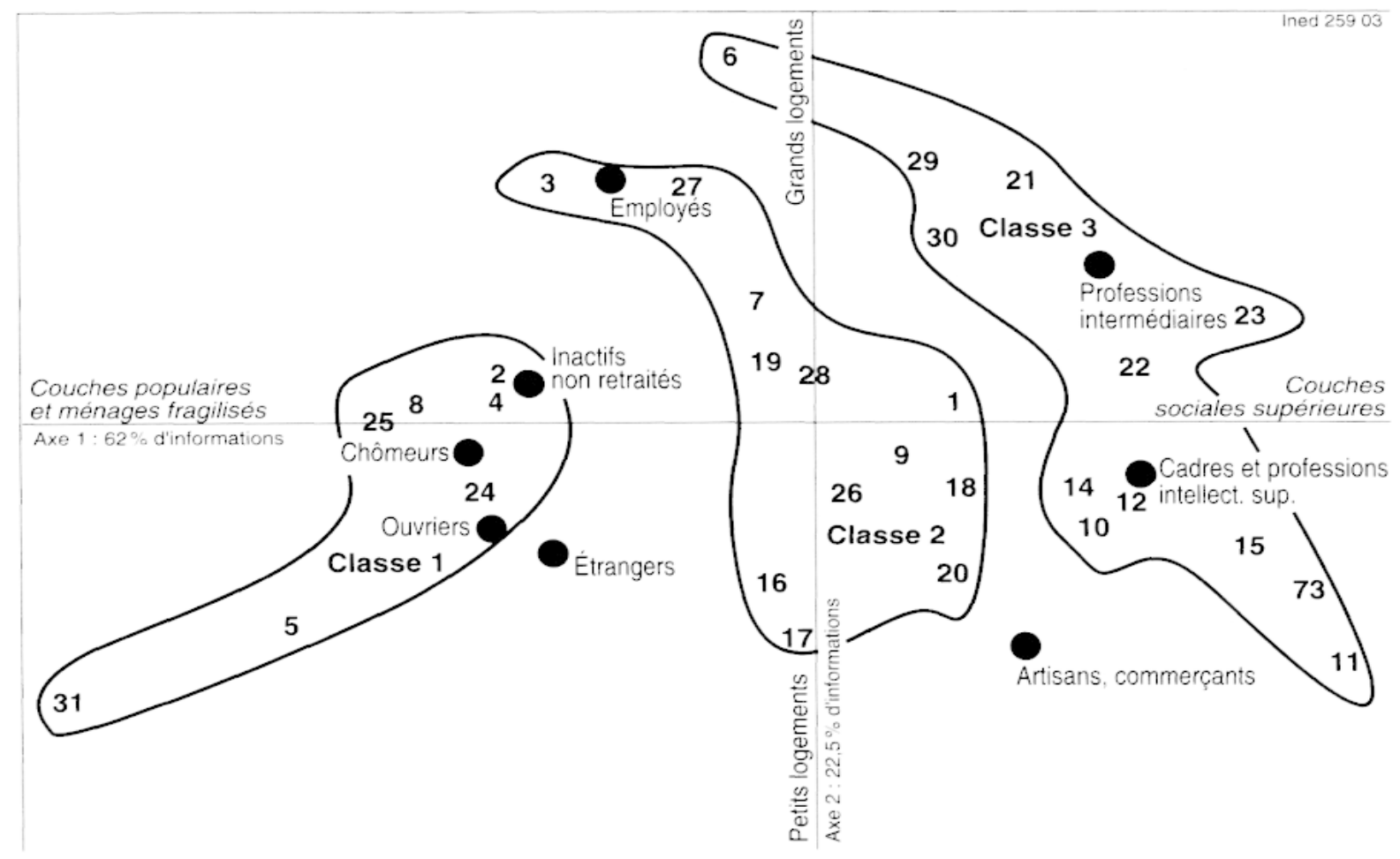

Figure 1.- Représentation des trois classes d'habitat sur le plan d'analyse factorielle

Note: les numéros renvoient aux types de logements définis au tableau 1.

Après avoir construit chacune des classes, il reste à les interpréter. La représentation des classes d'habitat sur le plan factoriel nous permet d'effectuer une première analyse de la hiérarchie des statuts sociorésidentiels départementaux (figure 1). Cette analyse est facilitée par le fait que le premier axe du plan traduit l'échelle des positions sociales des occupants des types de logements, allant des couches populaires et fragilisées (à gauche de l'axe) vers les couches sociales les plus élevées (à droite de l'axe). Ce constat montre que les ménages situés aux deux extrêmes de la hiérarchie sociale n'occupent pas les mêmes types de logements. À ce titre, on peut donc considérer que l'échelle des statuts sociorésidentiels épouse les hiérarchies de la société globale. Dans cette logique, la classe 1 regroupe les logements aux statuts les moins élevés, la classe 3 les logements aux statuts les plus élevés, tandis que la classe 2 se trouve dans une position intermédiaire entre ces deux extrêmes.

L'analyse factorielle et la classification hiérarchique nous permettent donc de regrouper qualitativement des types d'habitat en fonction des caractéristiques de leurs occupants et de les situer sur une échelle des statuts socio-résidentiels. Mais nous n'avons aucune idée de l'ampleur des distances qui séparent les positions des différents types de logements ou des classes d'habitat. De plus. l'objectif de cette recherche est, in fine, de mesurer et d'analyser les variations de ces positions dans l'espace, afin 


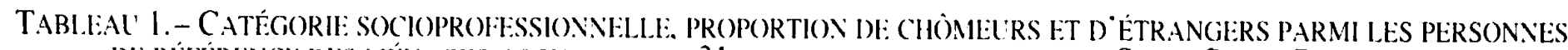

DE REFERE.VCE DES MENAGES OCCLPANT LES 31 TYPES DE LOGEMIENTS IE LA SEINE-S AINT-DENIS (EN \%) 


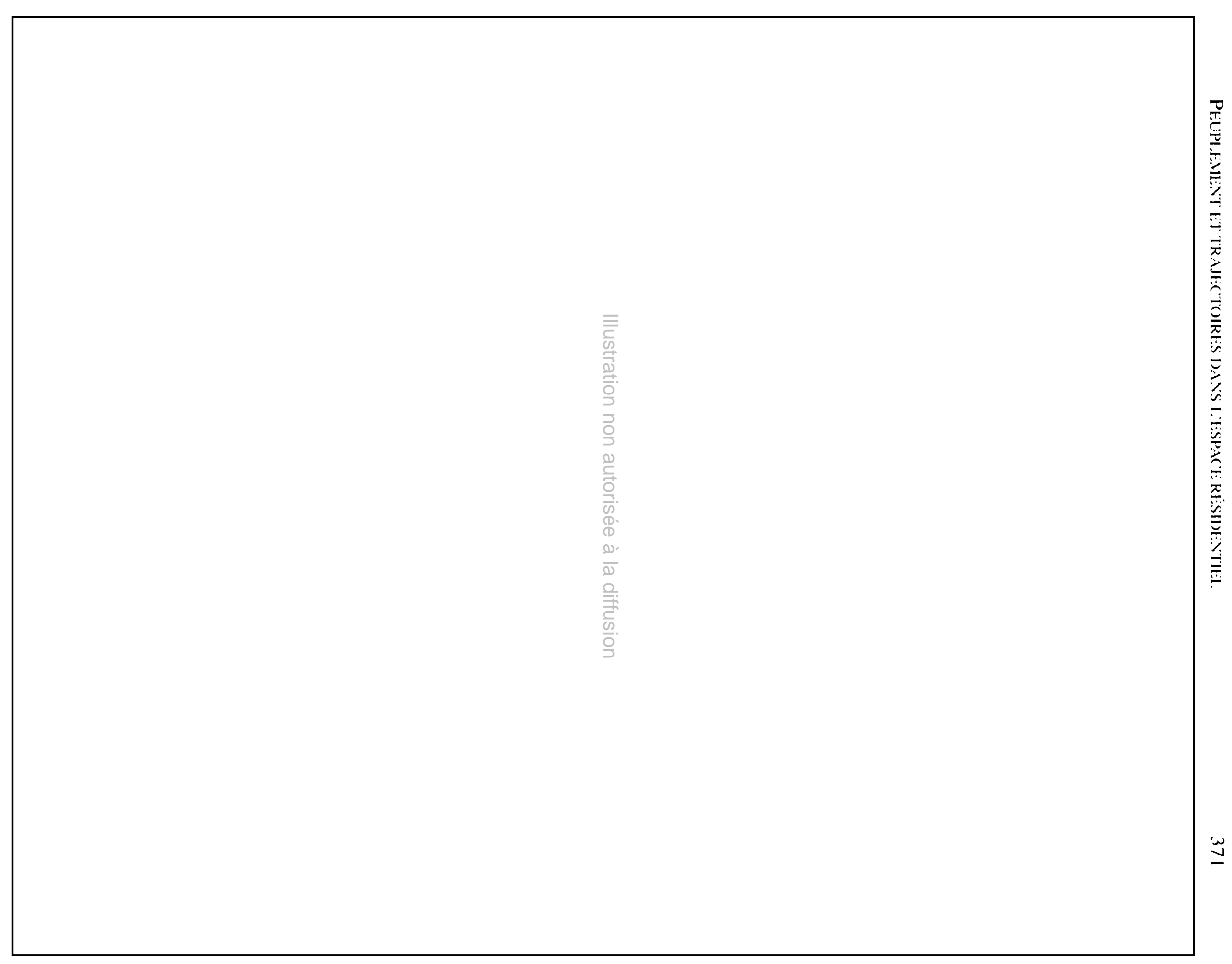


d'évaluer l'impact des marquages sociaux et des effets locaux sur l'organisation générale du peuplement des différents types de logements. Pour y parvenir, il est donc nécessaire de traduire quantitativement les résultats de cette première analyse.

En nous appuyant sur les résultats précédents, nous avons donc interprété les statuts socio-résidentiels des types de logements et des classes d'habitat à partir d'un indicateur quantitatif. Pour le construire, nous avons considéré que les ménages qui occupent les meilleures positions dans la société globale sont aussi ceux qui ont les meilleurs statuts sociorésidentiels dans l'espace local. Pour chacun des types de logements, l'indicateur rapporte les proportions de personnes de référence des ménages qui sont cadres ou exercent une profession intermédiaire (considérées comme révélatrices d'un statut socio-résidentiel élevé du logement) aux proportions d'étrangers et/ou de chômeurs (considérées comme révélatrices d'un statut socio-résidentiel bas du type de logement). L'indice est ensuite rapporté à la valeur du taux pour l'ensemble du département ${ }^{(1)}$ (tableau 2).

Une valeur de l'indice proche de 0 indique que le type de logement a un statut socio-résidentiel bas : ses occupants se caractérisent par une surreprésentation des étrangers et des chômeurs et une sous-représentation des cadres et des professions intermédiaires par rapport à l'ensemble de la population du département.

Une valeur de l'indice égale ou proche de 1 indique un statut sociorésidentiel moyen, c'est-à-dire que les catégories supérieures et les ménages fragilisés sont en proportion identique ou voisine dans le type de logement et dans le département.

Plus la valeur de l'indice s'accroît au-delà de 1 , et plus cela indique un statut socio-résidentiel élevé du type de logement : ses occupants se caractérisent par une surreprésentation des cadres et des professions intermédiaires et une sous-représentation des étrangers et des chômeurs par rapport à l'ensemble de la population du département.

(1) Cet indice mesure en fait l'écart entre la proportion de ménages appartenant aux catégories sociales supérieures et ceux appartenant aux catégories fragilisées dans le type de logement $j$. II a le mérite de la simplicité et s'apparente à l'indice, devenu classique, de dissimilarité proposé par les sociologues nord-américains dans les années 1950 (Duncan et Duncan, 1955). Il s'en distingue entre autres par le fait qu'il ne varie pas entre 0 et 1 . Il aurait été envisageable d'utiliser l'indice de dissimilarité pour calculer les indices de statut socio-résidentiel, mais il présente l'inconvénient de ne pas spécifier les catégories de populations majoritaires ou minoritaires dans le secteur étudié (la variation entre deux populations est présentée en valcur absolue). Cet obstacle aurait pu être facilement levé en proposant un indice variant entre +1 et -1 . Mais dans ce cas, il fallait abandonner la pondération par la valeur globale de l’indice pour le département. Or, celle-ci nous semblait importante dans la mesure où elle peut permettre d'effectuer ultérieurement des comparaisons entre sites. en éliminant les effets structurels des caractéristiques de la population globale sur loccupation de chacun des types de logements (Lévy et Haumont, 2001 ; Lévy, 2002). Cette pondération introduit en outre une indication supplémentaire sur l'écart entre la spécialisation sociale de chacun des types de logements et celle de la population globale du département. Pour ces différentes raisons, nous avons préféré maintenir le calcul de l'indice socio-résidentiel avec la formule proposée, en ayant conscience de ses faiblesses, notamment du fait qu il n’a pas de borne supéricure et que ses limites seraient même inexistantes dans le cas (peu probable) où il n'y aurait aucun chômeur et aucun étranger parmi les occupants d'un type de logement. 
TABIFAL 2. - INDICLS DU STATUT SOCIO-RÉSIDENTIEL DES 31 TYPES DE LOGEMENT DE LA SEINIE-SAINT-DENIS

\begin{tabular}{|c|c|c|c|c|c|c|}
\hline \multirow[t]{2}{*}{$\begin{array}{c}\text { Type de } \\
\text { logement (1) }\end{array}$} & $\begin{array}{c}\text { Proportion } \\
\text { de cadres } \\
\text { et prof. intel. } \\
\text { sup. } \\
\%\end{array}$ & $\begin{array}{c}\text { Proportion } \\
\text { de prof. } \\
\text { interm. } \\
\%\end{array}$ & $\begin{array}{c}\text { Proportion } \\
\text { d'étrangers } \\
\qquad \%\end{array}$ & $\begin{array}{c}\text { Proportion } \\
\text { de chomeurs } \\
\%\end{array}$ & Indice brut & Indice pondéré \\
\hline & {$[\mathrm{a}]$} & [b] & {$[\mathrm{c}]$} & [d] & {$[e]=[a+b] /[c+d]$} & {$[\mathrm{f}]=[\mathrm{e}] /[1,3]$} \\
\hline 1 & 17,0 & 25,1 & 17,3 & 7,0 & 1,7 & 1,3 \\
\hline 2 & 3,5 & 16,8 & 16,7 & 13,3 & 0,7 & 0,5 \\
\hline 3 & 3,3 & 18,6 & 11,2 & 11,6 & 1,0 & 0,7 \\
\hline 4 & 4,0 & 15,7 & 20,5 & 10,8 & 0,6 & 0,5 \\
\hline 5 & 5,2 & 10,7 & 42,4 & 12,2 & 0,3 & 0,2 \\
\hline 6 & 7,8 & 26,1 & 7,9 & 8,4 & 2,1 & 1,6 \\
\hline 7 & 8,8 & 22,7 & 15,9 & 7,9 & 1,3 & 1,0 \\
\hline 8 & 3,5 & 14,9 & 16,3 & 13,3 & 0,6 & 0,5 \\
\hline 9 & 9.1 & 21.4 & 9,1 & 9,1 & 1,7 & 1,3 \\
\hline 10 & 14,5 & 25,3 & 9,5 & 5,7 & 2,6 & 2,0 \\
\hline 11 & 34,6 & 21,0 & 10,5 & 3,4 & 4,0 & 3,1 \\
\hline 12 & 15,4 & 27,0 & 8,6 & 5,2 & 3,0 & 2,3 \\
\hline 13 & 27,9 & 23,6 & 10,0 & 4,2 & 3,6 & 2,8 \\
\hline 14 & 14,6 & 25,6 & 13,9 & 3,8 & 2,3 & 1,7 \\
\hline 15 & 24,0 & 26,3 & 15,1 & 3,1 & 2,8 & 2,1 \\
\hline 16 & 10,4 & 17,8 & 10,1 & 11,2 & 1,3 & 1,0 \\
\hline 17 & 11,7 & 18,3 & 18,5 & 10,2 & 1,0 & 0,8 \\
\hline 18 & 15,4 & 21,2 & 15,5 & 7,0 & 1,6 & 1,2 \\
\hline 19 & 9,7 & 21,7 & 18,4 & 8,4 & 1,2 & 0,9 \\
\hline 20 & 16,5 & 21,5 & 20,9 & 6,7 & 1,4 & 1,1 \\
\hline 21 & 15,6 & 28,3 & 7,6 & 5,7 & 3,3 & 2,5 \\
\hline 22 & 21,3 & 29,0 & 9,7 & 5,0 & 3,4 & 2,6 \\
\hline 23 & 26,5 & 30,5 & 9,4 & 3,5 & 4,4 & 3,4 \\
\hline 24 & 7,1 & 16,9 & 20,2 & 11,6 & 0,8 & 0,6 \\
\hline 25 & 6,0 & 17,9 & 31,1 & 13,5 & 0,5 & 0,4 \\
\hline 26 & 12,1 & 22.8 & 22,0 & 8,3 & 1,2 & 0,9 \\
\hline 27 & 9,3 & 25,7 & 15,4 & 8,8 & 1,4 & 1,1 \\
\hline 28 & 11,7 & 24,4 & 18,2 & 8.0 & 1,4 & 1,1 \\
\hline 29 & 12,9 & 29,3 & 11,9 & 8,0 & 2,1 & 1,6 \\
\hline 30 & 18,3 & 24,7 & 15,0 & 6,5 & 2,0 & 1,5 \\
\hline 31 & 2,3 & 9,3 & 43,9 & 18.0 & 0,2 & 0,1 \\
\hline \multicolumn{7}{|c|}{ Ensemble de la Seine-Saint-Denis } \\
\hline & 11,8 & 21,6 & 17,1 & 8,5 & 1,3 & 1,0 \\
\hline
\end{tabular}

Le même calcul est effectué pour chacune des classes d'habitat. On dispose ainsi d'une représentation des types de logements dans le département, et l'indice nous permet de situer chacune des classes en fonction des statuts socio-résidentiels de l'habitat de la Seine-Saint-Denis (tableau 3). 
TABIFAL 3. - TYPLS DE LOOEMENT, (I.ASSES D'HABITAT ITT LILLRS INDICES DE STATUT SOCIO-RÉSIDENTIEI.

DANS LEE DEPARTEMENT DE I.A SIIINE-SAINT-DINIS

\begin{tabular}{|c|c|c|c|}
\hline 31 types de logements & $\begin{array}{l}\text { Indice pondéré du statut } \\
\text { socio-résidentiel }\end{array}$ & 3 classes d'habitat & $\begin{array}{l}\text { Indice pondéré du statut } \\
\text { socio-résidentiel }\end{array}$ \\
\hline $\begin{array}{l}\text { 2. Habitat social avec confort construit avant } 1949 \\
\text { 4. Habitat locatif social avec confort construit de } 1949 \text { à } 1974 \text { de } 3 \text { ou } 4 \text { pièces } \\
\text { 5. Habitat locatif social avec confort construit de } 1949 \text { à } 1974 \\
\text { de } 5 \text { pièces ou plus } \\
\text { 8. Habitat locatif social sans confort } \\
\text { 24. Habitat collectif privé en accession à la propriété sans confort } \\
\text { 25. Habitat collectif privé en location avec confort construit avant } 1949 \\
\text { de } 1 \text { ou } 2 \text { pièces } \\
\text { 31. Habitat collectif privé en location sans confort }\end{array}$ & $\begin{array}{l}0.5 \\
0.5 \\
0.2 \\
0.5 \\
0.6 \\
0.4 \\
0.1\end{array}$ & $\begin{array}{l}\text { Logements au statut } \\
\text { socio-résidentiel bas }\end{array}$ & 0.4 \\
\hline $\begin{array}{l}\text { 1. Accession aidée } \\
\text { 3. Habitat locatif social avec confort construit de } 1949 \text { à } 1974 \text { de } 1 \text { ou } 2 \text { pièces } \\
\text { 7. Habitat locatif social avec confort construit depuis } 1975 \text { de } 3 \text { pièces ou pius } \\
\text { 9. Habitat individuel privé en accession à la propriété avec confort construit } \\
\text { avant } 1949 \text { de } 1 \text { ou } 2 \text { pièces } \\
\text { 16. Habitat individuel privé en accession à la propriété sans confort } \\
\text { 17. Habitat individuel privé en location construit avant } 1949 \\
\text { 18. Habitat individuel privé en location construit depuis } 1949 \\
\text { 19. Habitat collectif privé en accession à la propriété avec confort construit } \\
\text { avant } 1949 \text { de } 1 \text { ou } 2 \text { pièces } \\
\text { 20. Habitat collectif privé en accession à la propriété avec confort construit } \\
\text { avant } 1949 \text { de } 3 \text { pièces ou plus } \\
\text { 26. Habitat collectif privé en location avec confort construit avant } 1949 \\
\text { de } 3 \text { pièces ou plus } \\
\text { 27. Habitat collectif privé en location avec confort construit de } 1949 \text { à } 1974 \\
\text { de } 1 \text { ou } 2 \text { pièces } \\
\text { 28. Habitat collectif privé en location avec confort construit de } 1949 \text { à } 1974 \\
\text { de } 3 \text { pièces ou plus }\end{array}$ & $\begin{array}{l}1.3 \\
0.7 \\
1.0 \\
1.3 \\
1.0 \\
0.8 \\
1.2 \\
0.9 \\
1.1\end{array}$ & $\begin{array}{l}\text { Logements au statut } \\
\text { socio-résidentiel moyen }\end{array}$ & 1.0 \\
\hline
\end{tabular}




\begin{tabular}{|c|c|c|c|}
\hline 31 types de logements & $\begin{array}{l}\text { Indice pondéré du statut } \\
\text { socio-résidentiel }\end{array}$ & 3 classes d’habitat & $\begin{array}{l}\text { Indice pondéré u statut } \\
\text { socio-résidentiel }\end{array}$ \\
\hline 6. Habitat locatif social avec confort construit depuis 1975 de 1 ou 2 pièces & 1,6 & & \\
\hline $\begin{array}{l}\text { 10. Habitat individuel privé en accession à la propriété avec confort construit } \\
\text { avant } 1949 \text { de } 3 \text { ou } 4 \text { pièces }\end{array}$ & 2.0 & & \\
\hline $\begin{array}{l}\text { 11. Habitat individuel privé en accession à la propriété avec confort construit } \\
\text { avant } 1949 \text { de } 5 \text { pieces ou plus }\end{array}$ & 3,1 & & \\
\hline $\begin{array}{l}\text { 12. Habitat individuel privé en accession à la propriété avec confort construit } \\
\text { de } 1949 \text { à } 1974 \text { de } 1 \text { à } 4 \text { pièces }\end{array}$ & 2.3 & & \\
\hline $\begin{array}{l}\text { 13. Habitat individuel privé en accession à la propriété avec confort construit } \\
\text { de } 1949 \text { à } 1974 \text { de } 5 \text { pièces ou plus }\end{array}$ & 2.8 & & \\
\hline $\begin{array}{l}\text { 14. Habitat individuel privé en accession à la propriété avec confort construit } \\
\text { depuis } 1975 \text { de } 1 \text { à } 4 \text { pièces }\end{array}$ & 1,7 & & \\
\hline $\begin{array}{l}\text { 15. Habitat individuel privé en accession à la propriété avec confort construit } \\
\text { depuis } 1975 \text { de } 5 \text { pièces ou plus }\end{array}$ & 2,1 & socio-résidentiel élevé & 2.3 \\
\hline $\begin{array}{l}\text { 21. Habitat collectif privé en accession à la propriété avec confort construit } \\
\text { de } 1949 \text { à } 1974 \text { de } 1 \text { ou } 2 \text { pièces }\end{array}$ & 2.5 & & \\
\hline $\begin{array}{l}\text { 22. Habitat collectif privé en accession à la propriété avec confort construit } \\
\text { de } 1949 \text { a } 1974 \text { de } 3 \text { pieces ou plus }\end{array}$ & 2,6 & & \\
\hline $\begin{array}{l}\text { 23. Habitat collectif privé en accession à la propriété avec confort construit } \\
\text { depuis } 1975\end{array}$ & 3,4 & & \\
\hline $\begin{array}{l}\text { 29. Habitat collectif privé en location avec confort construit depuis } 1975 \\
\text { de } 1 \text { ou } 2 \text { pièces }\end{array}$ & 1.6 & & \\
\hline $\begin{array}{l}\text { 30. Habitat collectif privé en location avec confort construit depuis } 1975 \\
\text { de } 3 \text { pièces ou plus }\end{array}$ & 1.5 & & \\
\hline
\end{tabular}

Source : calculs de l'auteur d'après les données du recensement de la population de 1990 
La hiérarchisation en trois classes permet de bien représenter l'organisation du peuplement dans l'habitat de la Seine-Saint-Denis. On constate une bonne correspondance entre la valeur de l'indice des classes et celles des indices des types de logements qui les composent (tableau 3). Les distances entre les positions extrêmes sont importantes. La valeur de l'indice, pour le type de logement dont le statut est le plus bas est de 0,14 ; elle est de 3,4 pour le type de logement dont la position est la plus haute, soit un rapport de 1 à 24 entre les extrêmes. Cet écart se réduit lorsque les logements sont regroupés en classes de statut socio-résidentiel, mais il reste tout de même marqué : il va de 1 à 6 entre la classe d'habitat au statut socio-résidentiel bas $(0,4)$ et la classe d'habitat au statut sociorésidentiel élevé $(2,3)$.

Par ailleurs, les classes d'habitat réunissent des types de logements aux caractéristiques physiques très différentes. Selon la date de construction des immeubles ou le nombre de pièces, les logements sociaux peuvent polariser les ménages fragilisés (types $2,4,5$ et 8 ), ou bien les ménages appartenant aux catégories supérieures (type 6). Le parc en accession à la propriété est quant à lui éclaté entre des habitats au statut socio-résidentiel bas (type 24), moyen (types 1, 9, 16,19 et 20) et élevé (types 10 à 15, 21 à 23). Et il en va de même pour les logements locatifs du parc privé. Cet éclatement confirme que, pour étudier l'impact de l'habitat sur le peuplement, on ne peut comparer a priori des classifications basées sur les statuts d'occupation, la taille ou les modes de financement du logement. Ces classifications ne restituent que partiellement les logiques de distribution des ménages dans le parc immobilier et les liens entre les caractéristiques de l'habitat et celles des ménages.

\section{Les effets territoriaux : marquages sociaux et aires résidentielles}

Au même titre que le logement, certaines adresses sont plus recherchées que d'autres. Il en résulte que les marquages sociaux des territoires peuvent attirer ou au contraire repousser certaines catégories de population, ce qui, localement, peut modifier le statut socio-résidentiel des logements. Les classes d'habitat ont-elles le même statut socio-résidentiel dans l'ensemble du département de la Seine-Saint-Denis ou bien la distribution résidentielle des ménages s'adapte-t-elle aux micro-structures immobilières locales?

Pour tester la stabilité spatiale de la hiérarchie socio-résidentielle, nous avons calculé l'indice du statut socio-résidentiel des classes d'habitat à l'échelon des communes. Pour cela, nous avons rapporté, pour chacune des quarante communes de la Seine-Saint-Denis, la proportion des personnes de référence des ménages qui sont cadres ou professions intermédiaires à celle des personnes de référence étrangères et/ou au chômage parmi les occupants de chacune des trois classes d'habitat. Ce taux 
communal est ensuite rapporté à la valeur du taux départemental comme précédemment. Puis, à l'aide d'une classification hiérarchique, nous regroupons les communes pour lesquelles la valeur des indices obtenus pour chacune des trois classes est la plus proche. Nous obtenons ainsi trois aires résidentielles qui caractérisent des formes de peuplement particulières.

L’aire résidentielle valorisée regroupe les communes dans lesquelles les valeurs de l'indice des classes d'habitat sont supérieures à celles du département, ce qui traduit une attraction des catégories sociales supérieures et une répulsion des ménages les plus fragilisés.

L’aire résidentielle intermédiaire regroupe les communes dans lesquelles les valeurs de l'indice sont proches des moyennes du département, ce qui signifie qu'il n'existe pas de marquage social susceptible d'influencer l'occupation des classes d'habitat.

L’aire résidentielle dévalorisée regroupe les communes dans lesquelles les valeurs de l'indice des classes d'habitat sont inférieures à celles du département, ce qui signifie que les marquages sociaux attirent les ménages fragilisés et repoussent les catégories supérieures.

La figure 2 présente les indices moyens des statuts socio-résidentiels des communes qui composent chacune des aires résidentielles. Elle met en évidence l'existence de marquages sociaux qui peuvent aboutir à moduler sensiblement la présence des catégories supérieures ou des ménages fragilisés parmi les occupants des différentes classes d'habitat. Dans ce cadre méthodologique, il devient possible de comparer ce qui est comparable, c'est-à-dire des groupes de logements au statut similaire, mais localisés dans des espaces distincts. Quatre points méritent d'être soulignés.

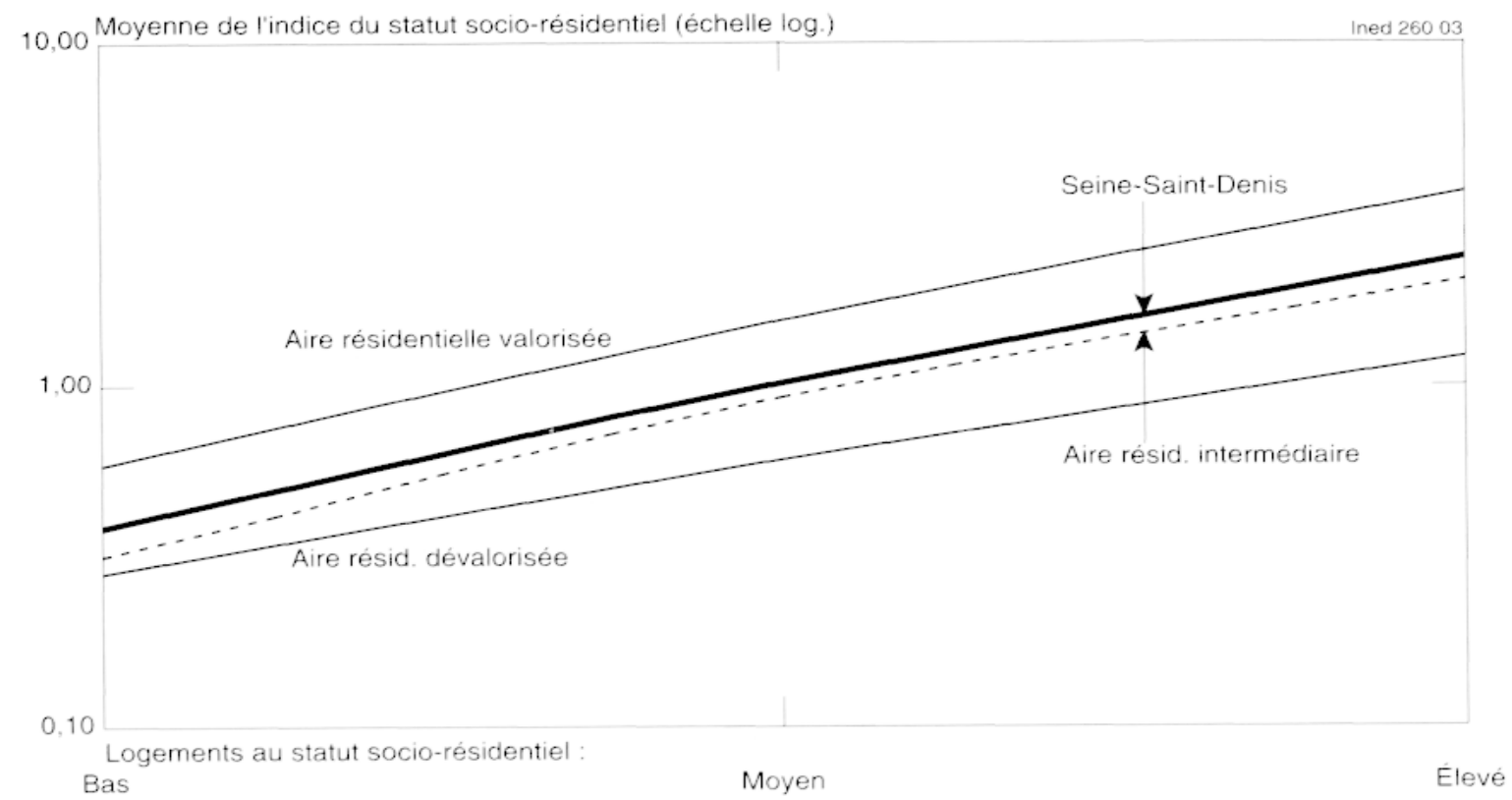

Figure 2.- Indices moyens des statuts socio-résidentiels selon les aires résidentielles de la Seine-Saint-Denis 
Tout d'abord, la méthode permet de comparer l'organisation hiérarchique - en quelque sorte la " pyramide socio-résidentielle »-interne à chacune des aires. La figure 2 illustre parfaitement la stabilité de cette organisation. Chacune des aires résidentielles présente une organisation hiérarchique conforme à celle du département, et la position de chaque classe d'habitat est identique dans chacune des hiérarchies communales. En d'autres termes, les marquages sociaux des communes ne remettent pas forcément en cause l'existence d'une hiérarchie propre à l'ensemble des communes du département.

Ensuite, les valeurs des indices permettent de mesurer l'impact de ces marquages sociaux sur les positions des classes d'habitat dans l'espace. Il devient alors possible de situer les positions d'une même classe d'habitat soumise à des marquages sociaux variables selon la localisation des logements. Ces positions mettent en évidence l'instabilité spatiale du statut de chacune des classes d'habitat. Par exemple, si l'on considère la position de la classe d'habitat au statut socio-résidentiel bas et que l'on prend comme indice de référence sa valeur moyenne dans le département, le marquage social de l'aire dévalorisée réduit l'indice de cette classe de - 0,10 , le marquage social de l'aire résidentielle intermédiaire produit également un écart négatif de $-0,06$, tandis que le marquage social de l'aire valorisée accrô̂t l'indice moyen de la classe de $+0,19$. Ces écarts peuvent être considérés comme la mesure de l'effet du marquage social de chacune des aires sur la position socio-résidentielle de la classe d'habitat au statut le moins élevé.

Pour autant, et c'est notre troisième point, le marquage social d'une aire $n$ 'agit pas de façon homogène sur les différentes classes d'habitat. La méthode permet également d'évaluer ces effets différenciés, par exemple lorsque l'on mesure les distances qui séparent la classe d'habitat au statut bas de celle au statut élevé dans chacune des aires résidentielles. En Seine-Saint-Denis, ces écarts entre les valeurs de l'indice s'élèvent à 0,92 dans l'aire résidentielle dévalorisée, 1,70 dans l'aire résidenticlle intermédiaire et 3,06 dans l'aire résidentielle valorisée (pour un écart de 1,96 au niveau du département). Le marquage social agit doublement : il module à la fois les distances à l'intérieur d'une même classe d'habitat localisée dans des aires distinctes, et les distances entre des classes d'habitat différentes localisées dans la même aire résidentielle. En Seine-Saint-Denis. l'impact du marquage social sur les distances entre les classes d'habitat est proportionnel à la valorisation de l'aire résidentielle. Si l'on prolonge le raisonnement, on peut même constater que le marquage social a davantage d'impact sur les classes d'habitat au statut élevé (en allant de l'aire dévaloriséc vers l'aire valorisée, les écarts avec la moyenne départementale sont respectivement de $-1,14,-0,33,+1,29)$ que sur celles dont le statut est bas (respectivement $-0,1,-0,06$ et $+0,19$ ). Il en résulte que la localisation produit moins de distance socio-résidentielle entre les 
occupants des classes d’habitat au statut bas qu'entre les occupants des classes d’habitat au statut élevé.

Enfin, la valeur de l'indice permet de déterminer la direction de trajectoires résidentielles mettant en jeu à la fois le statut socio-résidentiel du logement et le marquage social de la commune : un déménagement d'un logement au statut bas vers un logement au statut moyen, par exemple, n’implique pas nécessairement une trajectoire ascendante dans l'espace résidentiel. En Seine-Saint-Denis, c'est notamment le cas des logements au statut moyen des aires valorisées (indice $=1.55$ ) qui ont une position supérieure à celle des logements au statut élevé des aires dévalorisées (indice $=1,20$ ) (figure 2). On peut aussi déterminer la direction de trajectoires résidentielles réalisées à l'occasion de déménagements entre des classes d'habitat identiques mais localisées dans des aires distinctes: ainsi. lorsque des ménages se dirigent des logements au statut bas des aires dévalorisées (indice $=0,28$ ) vers ceux des aires valorisées (indice $=(0.57)$, ils réalisent un parcours ascendant.

Dès lors, si l'on voit bien l'utilité de la méthode pour l’analyse comparative de l'habitat et du peuplement dans différents espaces, à ce stade de la démarche, nous n’avons pas d’informations sur le rôle de la structure du pare immobilier communal dans la production des marquages sociaux et des aires résidentielles.

\section{Les zones d'habitat et les effets locaux}

Les classes d’habitat ne sont pas réparties de façon homogène entre les différentes communes de la Seine-Saint-Denis. Selon la composition des pares immobiliers en proportion de chacune des trois classes, on peut déterminer les caractéristiques dominantes des structures de l'habitat des communes du département pour distinguer trois zones d'habitat :

- la zone d'habitat haut de gamme regroupe les communes dans lesquelles sont concentrés des logements appartenant à la classe d'habitat au statut socio-résidentiel élevé tandis que les logements au statut sociorésidentiel bas y sont rares:

- la zone d'habitat bas de gamme qualific les communes dans lesquelles la production de logements au statut socio-résidenticl bas a été historiquement concentrée, repoussant sur d autres secteurs du département la construction de logements au statut socio-résidentiel élevé;

- la zone d'habitat mixte correspond aux communes dans lesquelles nous trouvons une proportion proche de logements appartenant aux trois classes d habitat.

La géographie de la structure des pares immobiliers communaux en Seine-Saint-Denis est présentée dans la carte l. Les zones d habitat bas de gamme regroupent près de la moitié des communes du département. les zones d’habitat mixte réunissent une commune sur cinq et les zones 


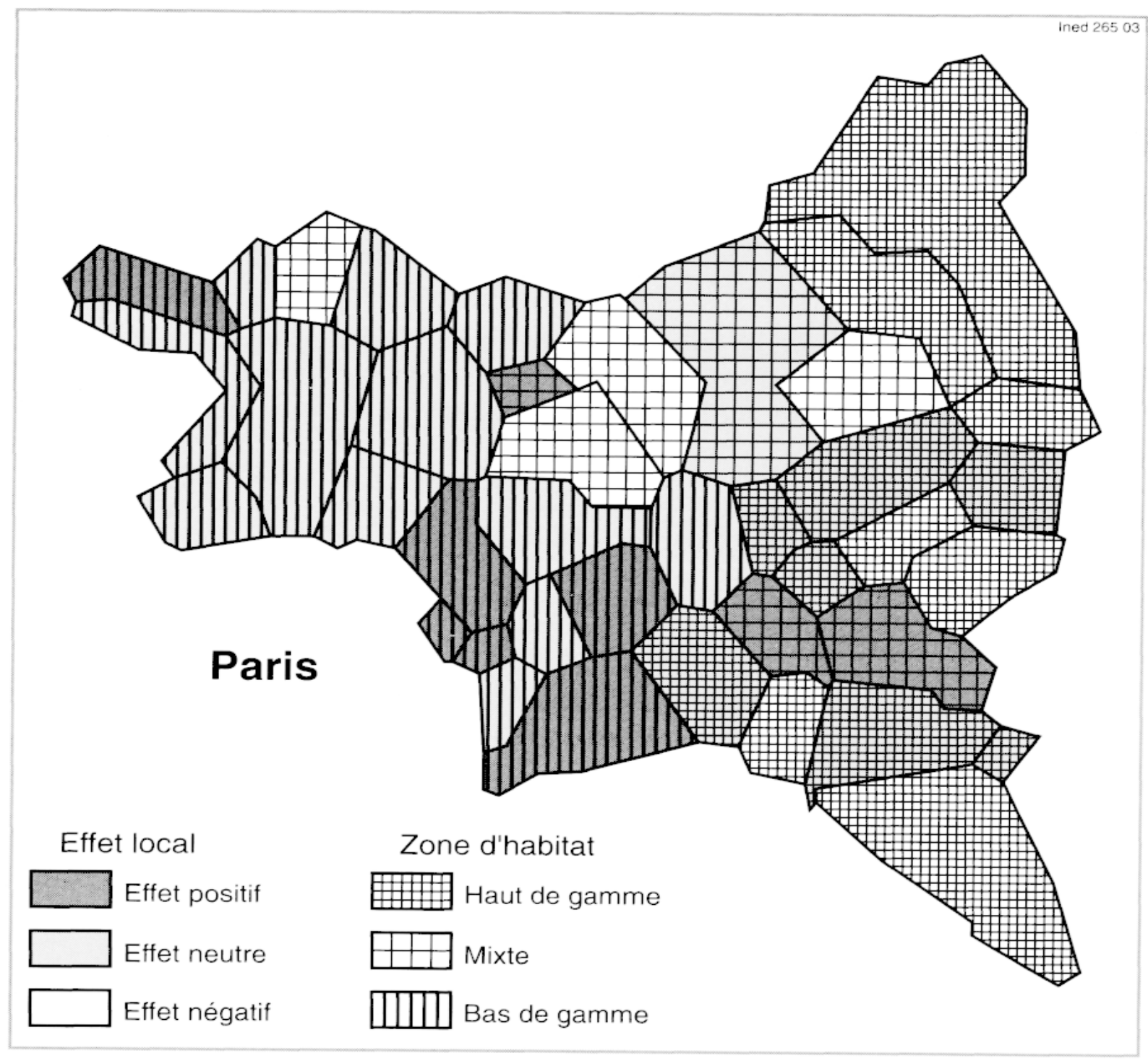

Carte 1.- Zones d'habitat et effets locaux dans les communes selon les aires résidentielles de la Seine-Saint-Denis

d'habitat haut de gamme représentent une commune sur trois. Sans entrer dans les détails, la structure du parc immobilier de la Seine-Saint-Denis est organisée selon une coupure est/ouest : à l'est, les zones d'habitat haut de gamme issues de la construction de lotissements pavillonnaires et d'un habitat ancien de qualité; à l'ouest, les zones d'habitat bas de gamme issues de la construction massive de logements sociaux et de l'existence d'immeubles ouvriers dégradés construits avant-guerre.

On peut penser que les caractéristiques dominantes d'une zone d'habitat influencent l'occupation de l'ensemble du parc immobilier du secteur, y compris celle des classes d'habitat les plus marginales. Si tel est le cas, une forte proportion de logements au statut socio-résidentiel élevé a 
pour conséquence un marquage social valorisant du peuplement de la commune et tire celui-ci vers le haut. À l'inverse, la concentration de logements au statut socio-résidentiel bas est un handicap pour la valorisation du secteur et ne favorise pas l'implantation des catégories sociales supérieures, y compris dans les fractions les plus valorisées du parc immobilier. Dans tous ces cas de figure, une zone d'habitat haut de gamme est synonyme d'aire résidentielle valorisée, une zone d'habitat mixte d'une aire résidentielle intermédiaire, et une zone d'habitat bas de gamme d'une aire résidentielle dévaloriséc. On dira alors que le peuplement du secteur ne subit pas d'effet local ou que son effet local est neutre.

Mais les associations entre zones d'habitat et aires résidentielles ne sont pas toujours aussi mécaniques. Le peuplement n'est pas systématiquement conforme à ce que la structure du parc et la hiérarchie des statuts socio-résidentiels dans l'agglomération pourraient laisser présager. Chacun a en mémoire des exemples de quartiers historiquement populaires attirant des couches moyennes, ou de quartiers anciennement bourgeois aujourd'hui déclassés. Dans ces cas, l'existence d'effets locaux explique les marquages sociaux à l'ouvre. Ces effets locaux peuvent être définis comme l'impact sur le peuplement de facteurs exogènes à la structure du parc (tels que la centralité d'un quartier ou d'une commune, sa position périphérique, son niveau d'équipement ou sa situation géographique qui rend très accessibles les quartiers attractifs par exemple). Ils nous permettent d'identifier les mécanismes de valorisation ou de dévalorisation agissant sur le peuplement des communes.

Pour situer les effets locaux, nous mesurons les écarts entre les positions attendues des statuts socio-résidentiels (définies par l'appartenance de la commune à une zone d'habitat) et les positions réelles (définies par l'appartenance de la commune à une aire résidentielle). La comparaison entre les caractéristiques de l'aire résidentielle et celles de la zone d'habitat à laquelle appartient la commune nous permet d'identifier, outre l'effet neutre déjà présenté, deux types d'effets locaux (tableau 4). Si l’on observe des marquages sociaux plus valorisés que ne le suggère la composition du pare immobilier, comme lorsqu une commune constitue une zone d'habitat bas de gamme mais une aire résidentielle valorisée, on dira alors

TABILAU 4. - DÉTIERINATION IJES IBHETS LOCALX

\begin{tabular}{|l|c|c|c|}
\hline & $\begin{array}{c}\text { Zone d'habitat } \\
\text { bas de gamme }\end{array}$ & $\begin{array}{c}\text { Zone d'habitat } \\
\text { mixte }\end{array}$ & $\begin{array}{c}\text { Zone d'habitat } \\
\text { haut de gamme }\end{array}$ \\
\hline $\begin{array}{l}\text { Aire résidentielle dévalorisée } \\
\text { Aire résidentielle intermédiaire }\end{array}$ & $=$ & - & - \\
Aire résidentielle valorisée & + & + & + \\
\hline $\begin{array}{l}\text { - : effet local négatif. } \\
\text { =: effet local neutre. } \\
+: \text { effet local positif. }\end{array}$
\end{tabular}


que la commune bénéficie d'un effet local positif. Si l'on constate au contraire des marquages sociaux moins valorisés que ne le suggère la composition du parc immobilier, par exemple lorsqu'une commune est une zone d'habitat mixte mais une aire résidentielle dévalorisée, on dira alors qu'elle subit un effet local négatif.

La carte 1 situe ces effets locaux dans le département. Elle montre que la structure du parc immobilier exerce une influence indéniable sur les formes de peuplement territoriales : la moitié des communes ne subissent pas d'effets locaux, ce qui signifie que leur marquage social relève de la composition de leur parc. Reste que, pour l'autre moitié, ce sont des facteurs autres que la structure de l'habitat qui permettent d'expliquer les marquages sociaux dans le peuplement : une commune sur quatre subit un effet local négatif tandis qu'une commune sur cinq bénéficie d'un effet local positif. Par définition, ces effets ne résultent pas de l'habitat et relèvent des structures sociales, de l'histoire ou des niveaux différenciés des services offerts par chacune des communes. Au-delà de ces spécificités locales, on peut cependant constater les effets de la situation géographique sur la valorisation du peuplement du département : sur les neuf communes bénéficiant d'un effet local positif, quatre sont limitrophes de la capitale.

Au terme de ces classifications. nous disposons donc de quatre types d'indicateurs : les statuts socio-résidentiels qui indiquent la position des logements et de leurs habitants dans l'espace résidentiel : les zones d'habitat qui caractérisent la composition des parcs immobiliers en fonction des statuts des logements; les aires résidentielles qui permettent d'identifier les effets des marquages sociaux sur le peuplement communal; et les effets locaux qui désignent les valorisations et dévalorisations du peuplement relevant des situations géographiques des communes (tableau 5).

Toute la question est done de comprendre comment les statuts sociorésidentiels des logements, la structure des parcs immobiliers, les marquages sociaux et les effets locaux sont appropriés par les ménages pour déterminer leurs choix résidentiels. Il s'agit également d'évaluer dans quelle mesure ces arbitrages participent à la constitution des peuplements dont nous venons d'évoquer les différentes formes.

\section{Logiques résidentielles et peuplements territoriaux}

Pour analyser les arbitrages résidentiels des ménages et leurs effets sur le peuplement, nous nous sommes appuyé sur les résultats d'une enquête auprès des ménages entrés dans un logement en Seine-Saint-Denis en 1994. L'échantillon de l'enquête a été constitué par un tirage aléatoire sur la totalité des logements dans lesquels avait été réalisée une ouverture de compteur d'électricité. Effectuée en 1995, elle a concerné 1568 mé- 
TABIFEIE 5. - PRF́SIENTATION SYNTHETIQL DE DES INDICATEL RS LTILISES

\begin{tabular}{|c|c|c|c|}
\hline Intitulé & Définition & Mode de calcul & Classification \\
\hline $\begin{array}{l}\text { Classes d'habitat } \\
\text { (statuts socio-résidentiels) }\end{array}$ & $\begin{array}{l}\text { Caractérisent la relation habitat/habitant définie comme } \\
\text { les liens symboliques qui associent les caractéristiques } \\
\text { du logement à la position de son occupant dans la } \\
\text { société globale ou locale. Ces liens confèrent au loge- } \\
\text { ment un statut socio-résidentiel identifié à partir des } \\
\text { associations entre les caractéristiques morphologiques } \\
\text { du parc immobilier et celles de ses occupants. }\end{array}$ & $\begin{array}{l}\text { Regroupement par classification hiérarchi- } \\
\text { que ascendante de } 31 \text { types de logements à } \\
\text { partir des caractéristiques sociales des per- } \\
\text { sonnes de référence des ménages occu- } \\
\text { pants. } \\
\text { Hiérarchisation par calcul d'un indice du } \\
\text { statut socio-résidentiel qui mesure les } \\
\text { écarts entre la proportion des ménages au } \\
\text { statut social élevé et des ménages sociale- } \\
\text { ment fragilisés parmi les occupants des } \\
\text { groupes d'habitat. }\end{array}$ & $\begin{array}{l}\text { Trois classes d'habitat : } \\
\text { - statut socio-résidentiel } \\
\text { élevé; } \\
\text { - statut socio-résidentiel } \\
\text { moyen: } \\
\text { - statut socio-résidentiel } \\
\text { bas. }\end{array}$ \\
\hline Zones d'habitat & $\begin{array}{l}\text { Identifient l'impact de la composition du pare immobi- } \\
\text { lier sur les représentations des marquages sociaux des } \\
\text { espaces résidentiels. Ce peut être par exemple l'impor- } \\
\text { tance de l'habitat de standing dans un quartier ou une } \\
\text { ville qui confêre à cette zone une image « chic ", ou à } \\
\text { l'inverse la présence d'un bâti ancien ou de logements } \\
\text { sociaux dégradés qui contribuent à donner à cet espace } \\
\text { une image "populaire". }\end{array}$ & $\begin{array}{l}\text { Regroupement des } 40 \text { communes du } \\
\text { département par classification hiérarchique } \\
\text { ascendante a partir de la proportion de } \\
\text { logements communaux appartenant à cha- } \\
\text { cune des trois classes d'habitat. }\end{array}$ & $\begin{array}{l}\text { Trois zones d'habitat } \\
\text { - haut de gamme: } \\
\text { - mixte: } \\
\text { - bas de gamme. }\end{array}$ \\
\hline Aires résidentielles & $\begin{array}{l}\text { Identifient les marquages sociaux réels des espaces } \\
\text { (quartier ou ville) qui, en influençant dans un sens ou } \\
\text { dans un autre les caractéristiques d'occupation des loge- } \\
\text { ments, modifient leurs statuts socio-résidentiels. C'est } \\
\text { notamment ce qui se produit lorsqu'un secteur histori- } \\
\text { quement populaire est soumis à une importante gentrifi- } \\
\text { cation, ou lorsqu'un espace peu à peu délaissé par les } \\
\text { couches moyennes se dévalorise. }\end{array}$ & $\begin{array}{l}\text { Calcul des indices des statuts socio- } \\
\text { résidentiels des trois classes d'habitat dans } \\
\text { chacune des } 40 \text { communes du départe- } \\
\text { ment. Regroupement en trois classes par } \\
\text { classification hiérarchique ascendante à } \\
\text { partir des combinaisons pour chaque com- } \\
\text { mune des valeurs des indices. }\end{array}$ & $\begin{array}{l}\text { Trois aires résidentielles : } \\
\text { - dévalorisée: } \\
\text { - intermédiaire: } \\
\text { - valorisée. }\end{array}$ \\
\hline Effets locaux & $\begin{array}{l}\text { Désignent les marquages sociaux ne relevant pas direc- } \\
\text { tement de la composition du pare immobilier. Ce sont } \\
\text { généralement des effets de localisation géographique } \\
\text { comme la centralité ou la position périphérique d'un } \\
\text { quartier ou d'une commune, ou bien le niveau d'équipe- } \\
\text { ment. }\end{array}$ & $\begin{array}{l}\text { Pour chacune des communes, mesure des } \\
\text { écarts entre les représentations des mar- } \\
\text { quages sociaux des espaces (zones d’habi- } \\
\text { tat) et les marquages sociaux réels (aires } \\
\text { résidentielles). Identifiés par une compa- } \\
\text { raison entre la zone d’habitat et l'aire } \\
\text { résidentielle. }\end{array}$ & $\begin{array}{l}\text { Trois effets locaux : } \\
\text { - positif; } \\
\text { - neutre; } \\
\text { - négatif. }\end{array}$ \\
\hline
\end{tabular}


nages, soit un ménage mobile en 1994 sur 32. Les ménages ont été interrogés sur leurs caractéristiques sociales et démographiques, leurs lieux de résidence et leurs logements actuel et antérieur. Les données obtenues ont été exploitées dans une triple direction : d'abord, pour cerner les trajectoires résidentielles des ménages; ensuite, pour mettre à jour les choix de localisation des ménages, les marges de manceuvre et les contraintes pesant sur leur décision; enfin, pour associer les logiques résidentielles des ménages et les évolutions des peuplements territoriaux à partir d'une observation des parcours résidentiels du moment.

Afin d'étudier les trajectoires résidentielles des ménages, nous disposons au terme de l'étude de l'habitat et du peuplement précédente de trois typologies. La première concerne les statuts socio-résidentiels des classes d'habitat : élevé (Élev.), moyen (Moy.) ou bas (Bas). La deuxième classe les communes en fonction de la structure locale de l'habitat : zone d'habitat haut de gamme (H), mixte (M) ou bas de gamme (B). La troisième, enfin, se réfère aux marquages sociaux et indique les effets locaux sur le peuplement : positif $(+)$, neutre $(=)$ ou négatif $(-)$. Au total, les mobilités résidentielles ont pu être observées pour douze catégories d'habitat qui tiennent compte du statut résidentiel, de la structure de l'habitat des communes et des effets locaux (tableau 6 ).

TABLEAL 6. - LISS DOL \%I: CATÉGORILS D’HABITAT

\begin{tabular}{|c|c|c|}
\hline Zones d'habitat & Classes d'habitat & Intitulé des catégories \\
\hline $\begin{array}{l}\text { Zones d'habitat mixte ou haut } \\
\text { de gamme sans effet local }\end{array}$ & $\begin{array}{l}\text { Statut socio-résidentiel élevé } \\
\text { Statut socio-résidentiel moyen } \\
\text { Statut socio-résidentiel bas }\end{array}$ & $\begin{array}{l}\mathrm{M} \& \mathrm{H}=/ \text { Élev. } \\
\mathrm{M} \& \mathrm{H}=/ \text { Moy. } \\
\mathrm{M} \& \mathrm{H}=/ \text { Bas }\end{array}$ \\
\hline $\begin{array}{l}\text { Zones d'habitat mixte ou haut } \\
\text { de gamme soumises à un effet } \\
\text { local négatif }\end{array}$ & $\begin{array}{l}\text { Statut socio-résidentiel élevé } \\
\text { Statut socio-résidentiel moyen } \\
\text { Statut socio-résidentiel bas }\end{array}$ & $\begin{array}{l}\text { M\&H-/Élev. } \\
\text { M\&H-/Moy. } \\
\text { M\&H-/Bas }\end{array}$ \\
\hline $\begin{array}{l}\text { Zones d'habitat bas de gamme } \\
\text { sans effet local }\end{array}$ & $\begin{array}{l}\text { Statut socio-résidentiel élevé } \\
\text { Statut socio-résidentiel moyen } \\
\text { Statut socio-résidentiel bas }\end{array}$ & $\begin{array}{l}\mathrm{B}=/ \text { Élev. } \\
\mathrm{B}=/ \text { Moy. } \\
\mathrm{B}=/ \text { Bas }\end{array}$ \\
\hline $\begin{array}{l}\text { Zones d'habitat bas de gamme } \\
\text { soumises à un effet local positif }\end{array}$ & $\begin{array}{l}\text { Statut socio-résidentiel élevé } \\
\text { Statut socio-résidentiel moyen } \\
\text { Statut socio-résidentiel bas }\end{array}$ & $\begin{array}{l}\text { B+/Élev. } \\
\text { B+/Moy. } \\
\text { B+/Bas }\end{array}$ \\
\hline
\end{tabular}

\section{Direction de la mobilité résidentielle}

En 1995, $10 \%$ des ménages résidant en Seine-Saint-Denis étaient entrés dans leur logement au cours de l'année précédente. Les ménages mobiles en provenance d'un autre département représentent un peu plus d'un nouvel occupant sur trois. L'embourgeoisement de ce département confronté à la crise ne peut provenir que de l'attraction de ménages aisés 
qui n'habitaient pas auparavant en Seine-Saint-Denis. Ces derniers se répartissent très inégalement entre les différentes zones d'habitat qui composent chacun des espaces résidentiels.

Le tableau 7 montre que ce sont les communes où domine l'habitat bas de gamme qui constituent la zone stratégique du renouvellement du peuplement de la Seine-Saint-Denis. Lorsqu elles s'embourgeoisent $(B+)$, elles attirent des ménages d'origine extérieure au département ( $42 \%$ des nouveaux emménagés dans l'année); lorsqu'elles sont ancrées dans la fragilisation $(B=)$, les dynamiques du renouvellement ne reposent que sur les mobilités résidentielles internes à la Seine-Saint-Denis $(72 \%$ des nouveaux emménagés). Quant aux zones dans lesquelles domine l'habitat mixte ou haut de gamme, les arrivants extérieurs représentent de l'ordre de trois nouveaux emménagés sur dix, sans que l'on relève de fortes variations selon le marquage social des communes.

TABIEAL 7.- ORIGINE DES NOLVEALX RMMÉNAGÉS FN SHINE-SAINT-DENIS EN 1994 SELON LES ZONES D`HABITAT (EN \%)

\section{Illustration non autorisée à la diffusion}

Quelle que soit la zone d'habitat, les ménages qui ne quittent pas la Seine-Saint-Denis réalisent généralement des mobilités de proximité : parmi les ménages mobiles à l'intérieur du département, $63 \%$ restent dans la même commune.

Le tableau 8 montre que, parmi les ménages mobiles restant en Seine-Saint-Denis, rares sont ceux qui s'installent dans une nouvelle aire résidentielle ou une nouvelle zone d'habitat. Ces mobilités circulaires illustrent les coupures socio-spatiales qui existent dans le département.

C'est à la fois dans les communes au marquage social dévalorisé et dans les zones d'habitat populaires qui s'embourgeoisent $(B+)$ que l'on trouve les taux de mobilité circulaire les plus importants (respectivement $85 \%$ et $79 \%$ ). Cela signifie que les mobilités internes à l'aire résidentielle ou à la zone d’habitat sont d'autant plus fortes que les écarts entre les communes se creusent sous les effets de l'embourgeoisement du peuplement de certains secteurs. D'un côté. les communes disposant des 
ressources les plus importantes s'embourgeoisent par l'arrivée d'une population extérieure (tableau 7). Elles stabilisent sur place les ménages les plus aisés, y compris lorsqu'ils changent de logement, et se ferment aux habitants des autres communes, notamment les plus pauvres. D'un autre côté, les communes habitées par les moins fortunés agissent comme des repoussoirs : elles ne parviennent plus à renouveler leur peuplement et à attirer d'autres catégories de ménages. La mobilité résidentielle locale est alors assurée par les franges les plus modestes de la population de ces communes, c'est-à-dire par ceux dont les revenus ne leur permettent pas de se diriger vers des secteurs dont le marquage social est plus valorisé. Ils sont alors contraints de circuler à l'intérieur des communes les moins valorisées du département (tableau 8). Les mobilités circulaires relèvent donc tout autant de processus « agrégatifs » d'ancrage des plus aisés au sein des communes qui s'embourgeoisent, que de processus ségrégatifs d'assignation territoriale dans les communes qui ne parviennent pas à se moderniser.

TABLI:AU 8. - TAUX DE MOBILITÉ CIRCULAIRE IDANS I.ES ENTRLEES INTERNES AU DEPARTEMENT IDE I.A SEINE-SAINT-DENIS SFI,ON I, AIRE RÉSIDHNTIEIA. ET LA ZONE D'HABITAT (EN \%)

Pour cerner plus précisément la nature de ces mouvements, nous avons observé parmi les mobilités internes à une même aire résidentielle (80) \% des mobilités internes à la Scine-Saint-Denis) la direction des passages d'une classe d'habitat à une autre. Nous avons distingué les mobilités neutres (déménagement vers une classe d'habitat au statut sociorésidentiel identique à celui de la classe précédente), les mobilités ascendantes (déménagement vers une classe d'habitat au statut socio-résidentiel supérieur à celui de la classe précédente) et les mobilités descendantes (déménagement vers une classe d'habitat au statut socio-résidentiel inférieur à celui de la classe précédente). 
Le tableau 9 montre que $47 \%$ des ménages qui sont mobiles à l'intérieur d'une même aire résidentielle réalisent un parcours neutre, et $24 \%$ réalisent un parcours descendant. Au total. ce sont donc plus de sept ménages mobiles sur dix qui ne s'inscrivent pas dans une trajectoire ascendante.

Les mobilités neutres, qui représentent les mouvements circulaires majoritaires dans le département. concernent toutes les catégories de logements et toutes les aires résidentielles. Leur intensité tend cependant à augmenter avec le niveau de valorisation de la commune : elles constituent $40 \%$ des mouvements internes à l'aire résidentielle dévalorisée et $5.5 \%$ des mouvements internes à l’aire résidentielle valorisée. Mais la signification de ces deux types de mouvements nest pas la même, car les classes d'habitat dans lesquelles s'inscrivent les mobilités neutres varient selon le marquage social des communes: le statut social du type d habitat est d'autant plus élevé que l'aire résidentielle dans laquelle le mouvement est effectué est clle-même valorisée. C’est ainsi que dans l'aire résidentielle dévalorisée, $46 \%$ des mobilités neutres sont réalisées dans la classe d’habitat au statut bas et $11 \%$ dans la classe d'habitat au statut élevé; à l'opposé, dans l'aire résidentielle valorisée, 42 des mobilités neutres concernent la classe d'habitat au statut élevé et à peine $13 \%$ la classe d'habitat au statut bas. Les trajectoires neutres traduisent donc un ancrage dans le parc à statut élevé lorsqu'elles sont réalisées dans les aires valorisées et un ancrage dans l'habitat bas de gamme lorsqu'elles sont effectuées dans les communes aux marquages sociaux les plus dévalorisés.

TABIFAC 9. - DIRECTION IDES TRAIEC TOIRES DISS MOBILITES CIRCULAIRLS DANS IIES AIRISS RESIDINTIELILES DIE I.A SEINI-SAINT-DENIS (IEN \%)

Les mobilités ascendantes représentent quant à elles $29 \%$ des mouvements internes à la Seine-Saint-Denis: elles sont plus fréquentes dans les aires résidentielles intermédiaires et plus rares dans les aires résidentielles valorisées. Les trajectoires descendantes constituent enfin un peu moins d'une mobilité sur quatre réalisée au sein du département. Elles 
existent dans toutes les aires résidentielles, mais c'est dans les secteurs les moins valorisés que leur intensité est la plus forte $(30 \%)$. Elles accentuent ainsi la déqualification résidentielle des ménages fragilisés et mobiles.

Au total, $80 \%$ des ménages mobiles à l'intérieur du département de la Seine-Saint-Denis déménagent au sein d'une même aire résidentielle, et parmi eux, sept sur dix réalisent une trajectoire résidentielle neutre ou descendante. Ces données illustrent parfaitement la prépondérance d'une mobilité cloisonnée, réalisée par des ménages en panne de promotion sociale qui s'accrochent à leur statut résidentiel, et motivée par des événements survenus dans le cycle de vie plutôt que par un objectif de promotion résidentielle.

Il y a donc coexistence entre un modèle de parcours résidentiel horizontal dominant (déménagement vers un type d'habitat de même valeur dans une zone de même valeur), voire descendant, et un modèle de parcours ascendant (amélioration du statut permise soit par un changement de zone, soit par un changement de classe d'habitat ou les deux conjointement). Ce constat incite à accorder à la mobilité résidentielle interne au département un rôle stratégique pour comprendre les processus de peuplement dans leurs dimensions spatiale et temporelle. Dans quelle mesure les mobilités qui s'effectuent à l'intérieur du département nous aident-elles à comprendre la façon dont les logiques résidentielles des habitants participent à la construction des hiérarchies des types d'habitat et des marquages sociaux des communes? En retour, comment ces hiérarchies structurentelles les mouvements résidentiels des groupes sociaux et participent-elles à la construction et à l'évolution spatialement différenciée du peuplement local?

\section{Les filières économiques de mobilité}

Pour étudier ces questions, nous avons observé les mouvements résidentiels de deux catégories clés de la population du département : les ménages jeunes et aisés et les ménages d'âge actif aux faibles revenus. Les premiers sont des ménages dont la personne de référence a moins de 30 ans, qui ont des revenus annuels par unité de consommation (UC) égaux ou supérieurs au quatrième quintile de revenus. Ils constituent $18 \%$ des entrants dans un logement du département en 1994, et $46 \%$ des ménages mobiles de ce groupe d'âges. Les seconds sont des ménages peu aisés dont la personne de référence a entre 30 et 49 ans. Leurs revenus annuels par unité de consommation sont inférieurs au deuxième quintile de revenus. Ce groupe constitue $23 \%$ des ménages mobiles du département et $44 \%$ des ménages mobiles de cette classe d'âges. Les deux catégories représentent donc un peu plus de quatre ménages mobiles sur dix. Outre leur contribution aux mouvements résidentiels, elles présentent l'avantage de se situer aux deux extrémités de la hiérarchie sociale. Elles sont suffisamment opposées dans leurs logiques résidentielles pour pouvoir illustrer 
aussi bien les effets de leur propre mobilité que ceux des catégories situées entre ces deux extrêmes.

La figure 3 présente les caractéristiques des entrants dans les classes d'habitat de chacune des zones du département en 1994. Pour déterminer le profil des ménages mobiles, nous avons croisé la proportion de ménages aisés parmi ceux dont la personne de référence a moins de 30 ans (en abscisse) avec la proportion de ménages « pauvres » parmi ceux dont la personne de référence est âgée de 30 à 49 ans (en ordonnée). À partir des proportions moyennes d'entrants issus de ces deux populations dans un logement du département, nous obtenons quatre cases qui permettent de visualiser les habitats spécialisés dans l'accueil des ménages aisés (jeunes et 30-49 ans aisés), ceux essentiellement ouverts aux ménages à faibles revenus (jeunes et 30-49 ans pauvres) et enfin, les habitats qui accueillent un large spectre de population aux revenus diversifiés (mixte).

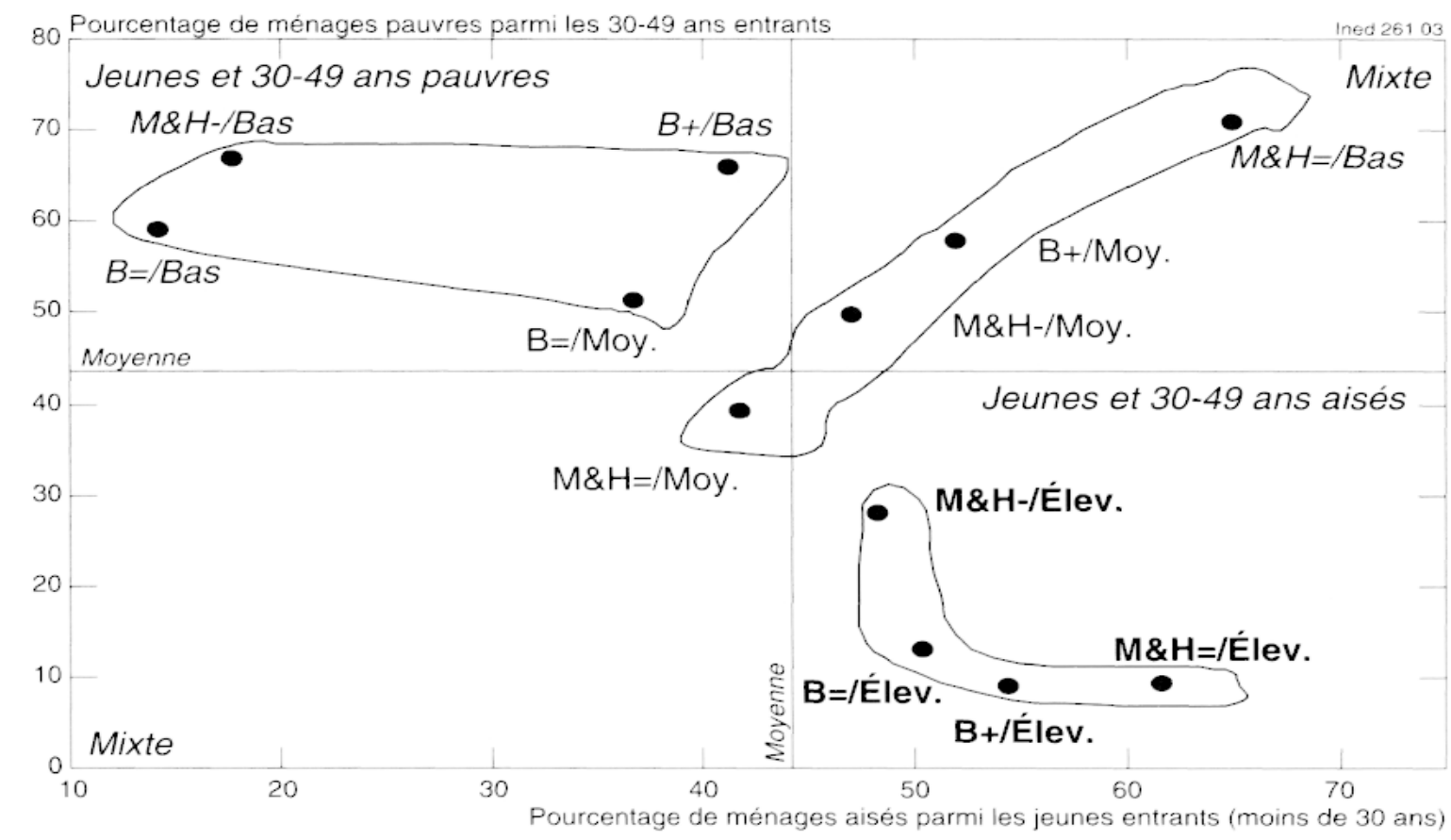

Figure 3.- Caractéristiques des ménages entrés dans les classes d'habitat de chacune des zones d'habitat du département de la Seine-Saint-Denis en 1994

Lecture : dans l'habitat bas de gamme situé dans la zone d'habitat mixte ou haut de gamme soumise à un effet local négatif (M\&H-/Bas) du département de la Seine-Saint-Denis, parmi les ménages entrés dans un logement en 1994 dont la personne de référence a moins de 30 ans, $20 \%$ ont des revenus élevés, et parmi les ménages dont la personne de référence a entre 30 et 49 ans, $67 \%$ ont des revenus faibles.

Les résultats obtenus à partir de ce croisement sont peu surprenants dans la mesure où les classes d’habitat apparaissent très spécialisées selon les revenus des ménages entrants. En d’autres termes, selon les revenus 
des ménages mobiles, les circuits résidentiels ne concernent pas les mêmes classes d'habitat et sont très cloisonnés. Ce constat traduit l'existence de trois filières économiques de mobilité résidentielle : une filière spécifique aux ménages modestes qui regroupe surtout les habitats à faible statut résidentiel (Bas) de l'ensemble des communes, située dans la case jeunes et 30-49 ans pauvres sur la figure 3; une filière spécialisée dans l'accueil des ménages aisés qui regroupe les habitats à haut statut résidentiel de l'ensemble des zones (Élev.), située dans la case jeunes et 3049 ans aisés; enfin, une troisième filière, située à la charnière des précédentes au centre de la figure, qui comprend l'essentiel des classes intermédiaires (Moy.) et concerne des ménages hétérogènes du point de vue de leur situation économique, des plus aisés aux plus pauvres.

Certains types d'habitat n'entrent pas dans ce modèle. C'est notamment le cas des logements au statut moyen localisés dans les zones d'habitat populaire stables ( $\mathrm{B}=/$ Moy.) et celui des logements au statut bas situé dans les zones d'habitat valorisé sans effet local ( $\mathrm{M} \& \mathrm{H}=/$ Bas). Globalement, les classes d'habitat situées aux deux extrêmes de la hiérarchie des statuts résidentiels sont cependant très spécialisées, les logements au statut moyen accueillant quant à eux des ménages aux revenus diversifiés. Il en résulte que les logements au statut socio-résidentiel moyen sont plus sensibles que les autres aux marquages sociaux des communes dans lesquelles ils sont localisés. Du fait de leur situation médiane entre l'habitat à haut et à bas statut résidentiel, ils jouent probablement un rôle majeur dans la diffusion des mécanismes de valorisation ou de dévalorisation du peuplement local.

\section{Parcours résidentiels du moment et cycle de vie familial}

Comment les statuts socio-résidentiels s'enchaînent-ils dans les parcours des ménages? Existe-t-il un statut propre à une ou plusieurs séquences du cycle de vie et du cursus résidentiel? Est-il possible de réaliser un parcours résidentiel complet dans une zone d'habitat, ou bien ces zones sont-elles spécialisées dans une ou plusicurs séquences du cursus résidentiel et du cycle de vie des ménages?

Afin d'identifier les trajectoires résidentielles des ménages à différentes périodes de leur cycle de vie, nous avons croisé la proportion de ménages constitués de personnes isolées ou en couple sans enfant avec celle des ménages dont la personne de référence a moins de 30 ans qui sont entrés dans chacune des classes d'habitat du département en 1994 (figure 4). Les quatre parties de la figure sont délimitées par les proportions moyennes observées pour ces deux groupes de ménages parmi les entrants dans un logement du département. La figure présente des modèles de parcours résidentiels orientés selon les enchaînements des principales séquences du cycle de vie : isolés et couples jeunes sans enfant correspon- 
dent à la séquence de formation du couple: familles jeunes et familles âgées traduisent les périodes de vie commune du couple et des enfants; isolés et couples âgés sans enfant représentent la période du « nid vide » où il ne reste que le couple après le départ des enfants.

Les logements appartenant à une même classe d'habitat (statut sociorésidentiel élevé, moyen ou bas) mais localisés dans les différentes zones du département ont été reliés sur la figure 4.

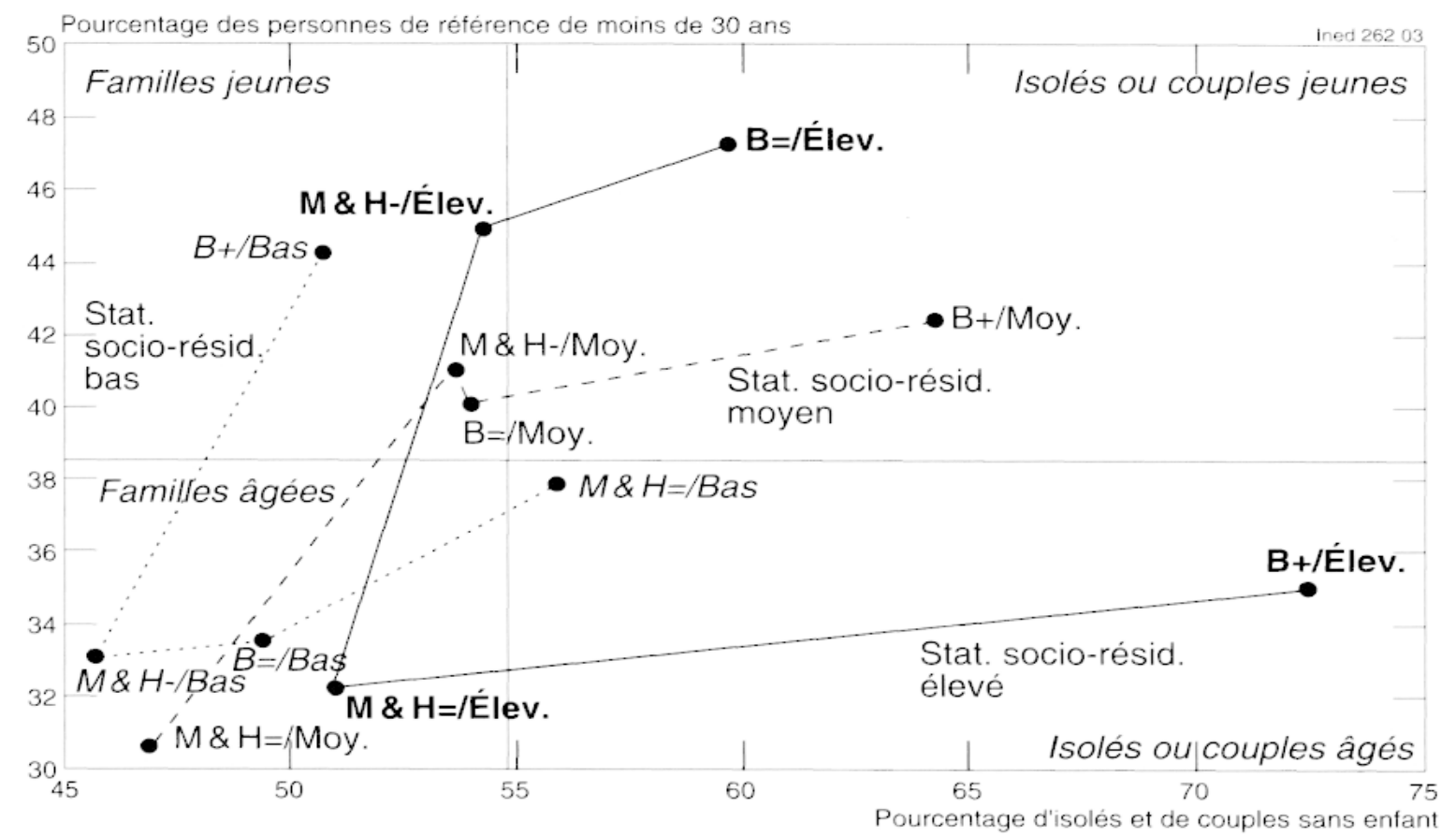

Figure 4. - Caractéristiques démographiques des ménages entrés dans les classes d'habitat du département de la Seine-Saint-Denis en 1994

Lecture : parmi les ménages entrés en 1994 dans un logement situé dans la classe d'habitat au statut socio-résidentiel élevé de la zone d'habitat bas de gamme sans effet local ( $B=/ E$ lev.) du département de la Seine-Saint-Denis, $47 \%$ des personnes de référence ont moins de 30 ans et $60 \%$ sont des personnes seules ou en couple sans enfant.

Globalement, la figure montre qu`il n’existe pas de spécialisation démographique des classes d'habitat, aucune d'entre elles n'étant regroupée dans une seule partic de la figure. Cela signifie qu'une même classe d'habitat peut être investie par des ménages engagés dans des périodes différentes du cycle de la vie familiale. Ce constat montre qu'il est tout à fait envisageable de réaliser plusieurs séquences du parcours résidentiel. voire un parcours résidentiel complet, en circulant entre des logements appartenant à une seule classe d’habitat. mais situés dans différentes zones de la Seine-Saint-Denis. Ces mouvements internes à une même classe d habitat constituent d’ailleurs près de la moitié des mobilités qui s'effectuent dans l'année dans le département. La caractéristique de la commune d’installa- 
tion (valorisée ou dévalorisée) devient alors une variable importante dans les critères de choix du logement, dans la mesure où la direction de la trajectoire résidentielle est dès lors déterminée par les marquages sociaux des communes de résidence successives.

Le regroupement des classes d'habitat à faible statut montre que si le parcours se réalise uniquement dans de tels logements, il est orienté des zones d'habitat bas de gamme embourgeoisé $(B+)$, soit vers celles ancrées dans la dévalorisation $(B=)$, soit vers des communes d'habitat mixte ou haut de gamme paupérisé $\left(M \& \mathrm{H}_{-}\right.$). La trajectoire résidentielle est alors descendante car, au fil des séquences du parcours, les marquages sociaux des communes d'installation sont de moins en moins valorisants. Une dernière séquence peut néanmoins être dirigée vers une zone haut de gamme sans effet local ( $M$ \& $H=$ ), mais elle concerne surtout les couples âgés sans enfant ou les isolés âgés.

À l'opposé, le regroupement des logements à statut élevé montre qu'un parcours s'inscrivant dans cette classe d'habitat débute soit dans des communes ancrées dans la fragilisation $(B=)$, soit dans des communes d'habitat mixte ou haut de gamme déqualifié ( $M \& H_{-}$), pour aboutir dans les zones d'habitat bas de gamme embourgeoisé $(B+)$ ou bien dans des communes d'habitat mixte ou haut de gamme le plus valorisé $(M \& \mathbf{H}=)$ : dans ce cas, les marquages sociaux successifs des communes d'installation permettent de qualifier les trajectoires d'ascendantes.

En d'autres termes, s'il est envisageable de réaliser un parcours résidentiel complet dans une même classe d'habitat dans le département de la Seine-Saint-Denis, seule l'inscription de ce parcours dans les logements à statut élevé favorise la réalisation d'une trajectoire résidentielle ascendante. Si ce n'est pas le cas, les ménages mobiles désireux d'améliorer leur position résidentielle doivent arbitrer en tenant compte du marquage social de la commune, mais aussi et surtout en déménageant dans des logements appartenant à différentes classes d'habitat.

Nous avons vu par ailleurs que les trois quarts des déménagements internes au département étaient réalisés entre des zones d'habitat identiques (tableau 8). Dans ce cas, la direction de la trajectoire résulte de l'enchaînement des statuts socio-résidentiels des logements successifs. La figure 4 montre que si l'on écarte les mobilités neutres, le modèle de parcours réalisé dans les zones d'habitat plutôt dévalorisées traduit une trajectoire descendante. Dans la zone d'habitat bas de gamme sans effet local $(\mathrm{B}=$ ) ou dans la zone d'habitat mixte ou haut de gamme subissant un effet local négatif ( $M \& H-$ ), le parcours est orienté des logements au statut élevé vers les logements au statut moyen puis les logements au statut bas. Mais les zones d'habitat les plus valorisées ne rentrent pas dans ce modèle de parcours. Dans la zone d'habitat bas de gamme bénéficiant d'un effet local positif $(B+)$, les trajectoires débutent dans les logements au statut bas ou moyen et se terminent dans les logements à statut socio-résidentiel élevé. Quant à la zone d'habitat mixte ou haut de gamme stabilisée dans la 
valorisation $(M \& H=)$, elle accueille uniquement des ménages en fin de parcours résidentiel, qu'il s’agisse de familles, de personnes isolées ou de couples âgés sans enfant.

L'exemple de la Seine-Saint-Denis montre donc que la pente de la trajectoire résidentielle dépend en grande partie du marquage social des communes d'installation. Dans ce cadre, l'adéquation entre le statut sociorésidentiel du logement et sa spécialisation démographique n’est ni mécanique, ni systématique. En conséquence, les logements au statut sociorésidentiel bas n'accueillent pas forcément des ménages en début de parcours résidentiel, pas plus que les logements au statut élevé n'ont vocation à accueillir des ménages en fin de parcours résidentiel. Ce constat conduit à s'interroger sur l'organisation des mobilités résidentielles dans les zones confrontées à la crise, dans une période où la promotion sociale au cours de la carrière professionnelle n'est plus assurée (Courgeau et Pumain, 1993 ; Chauvel, 1998). Par ailleurs, les ménages se fondent autant sur les statuts socio-résidentiels de l'habitat que sur les marquages sociaux et les effets locaux des communes pour déterminer leur trajectoire résidentielle.

\section{Renouvellement du peuplement et trajectoires résidentielles}

La prise en compte des revenus des ménages a mis en évidence l'existence de filières économiques de la mobilité. Celle de l'âge et de la taille des ménages a montré l'importance de l'enchaînement des marquages sociaux des communes pour déterminer le sens des trajectoires résidentielles. En croisant ces deux approches (les filières économiques et la direction des trajectoires au cours du cycle de vie), on peut maintenant s'interroger sur l'existence d'une organisation générale de la mobilité résidenticlle en Seine-Saint-Denis.

À partir des figures 3 et 4 , nous avons reconstitué les parcours résidentiels du moment des ménages aisés (revenus par UC supérieurs ou égaux au $4^{\circ}$ quintile de revenus) et des ménages aux faibles ressources (revenus par UC inférieurs au $2^{e}$ quintile). L'approche démographique est à nouveau mise en ceuvre sur la base des proportions de jeunes el d'isolés ou de couples sans enfant entrant dans chacune des classes d'habitat. Nous retrouvons donc les quatre séquences du cycle de vie d'un ménage.

Les figures 5 et 6 présentent une simulation des parcours réalisée à partir des informations concernant les caractéristiques des zones d'habitat et des ménages qui s’y installent. Insistons bien sur le fait qu il ne s’agit que d'un modèle construit sur les données du moment : rien ne nous permet d'aftirmer qu'un ménage situé à une étape du parcours en 1994 a connu les séquences précédentes et connaîtra les étapes futures présentées dans les figures, car celles-ci ont eu lieu. ou auront lieu. dans des contextes socio-économiques différents. Les parcours résidentiels du mo- 
ment présentent cependant l'intérêt de situer la mobilité dans le contexte actuel.

En ce qui concerne les ménages aisés (figure 5), différentes configurations sont envisageables selon que les ménages ne s'installent que dans l'habitat spécialisé dans d'accueil des catégories les plus favorisées (en gris foncé dans la figure 5), ou qu'ils se dirigent vers un habitat plus mixte socialement et économiquement (gris clair dans la figure 5). L’inscription dans une trajectoire ascendante n'est assurée qu'avec le premier type de parcours (flèches pleines), c'est-à-dire dans le cas où les séquences passent uniquement par des logements au statut socio-résidentiel élevé (Élev.). Le parcours débute alors dans la zone d'habitat bas de gamme sans effet local $(B=)$, se prolonge dans la zone mixte ou haut de gamme soumise à un effet local négatif ( $\mathrm{M} \& \mathrm{H}-$ ) lors de la deuxième séquence, puis soumise à un effet local neutre $(M \& H=)$ dans la troisième séquence, et se termine par une quatrième séquence dans la zone d'habitat bas de gamme embourgeoisé $(B+)$. Des zones d'habitat au marquage social de plus en plus valorisć sont donc investies au fur et à mesure de la progression dans le cursus résidentiel, et la territorialisation de la trajectoire permet ainsi aux ménages aisés qui se stabilisent dans le département de se construire une trajectoire ascendante tout en évoluant dans du logement haut de gamme.

Si les ménages aisés réalisent leur mobilité dans des habitats d'accueil d'une population aux revenus diversifiés (flèches en pointillé dans la figure 5), la pente ascendante de la trajectoire n'est plus garantie et devient fonction de l'enchaînement des zones et des types d'habitat lors des différentes séquences résidentielles, sachant qu'à n'importe quelle étape du parcours, il est toujours possible aux ménages de s'inscrire à nouveau dans la filière précédente. En ce sens, l'emménagement dans l'habitat d'accueil d'une population mixte (essentiellement composé de logements au statut moyen) peut apparaître comme une alternative au parcours résidentiel ascendant idéal typique des ménages les plus aisés du département. L'existence de telles trajectoires démontre également qu'un parcours ascendant n'est pas forcément linéaire et peut inclure des phases plus chaotiques (une première séquence descendante et les autres ascendantes par exemple).

Comme précédemment, l'existence d'un habitat qui accucille des populations aux revenus diversifiés est associée à différentes configurations quant à l'organisation des parcours résidentiels des ménages les moins aisés (figure 6). Mais contrairement aux cas de figure précédents, on ne peut envisager ici la réalisation d'un parcours résidentiel complet dans une seule classe d'habitat, la première séquence résidentielle passant nécessairement par un logement au statut moyen situé dans des secteurs d'habitat populaire qui s'embourgeoisent ( $B+/$ Moy.). Passé ce cap cependant, les autres séquences peuvent s'inscrire uniquement dans l'habitat bas de gamme (flèches pleines), notamment si elles ne passent que par des 


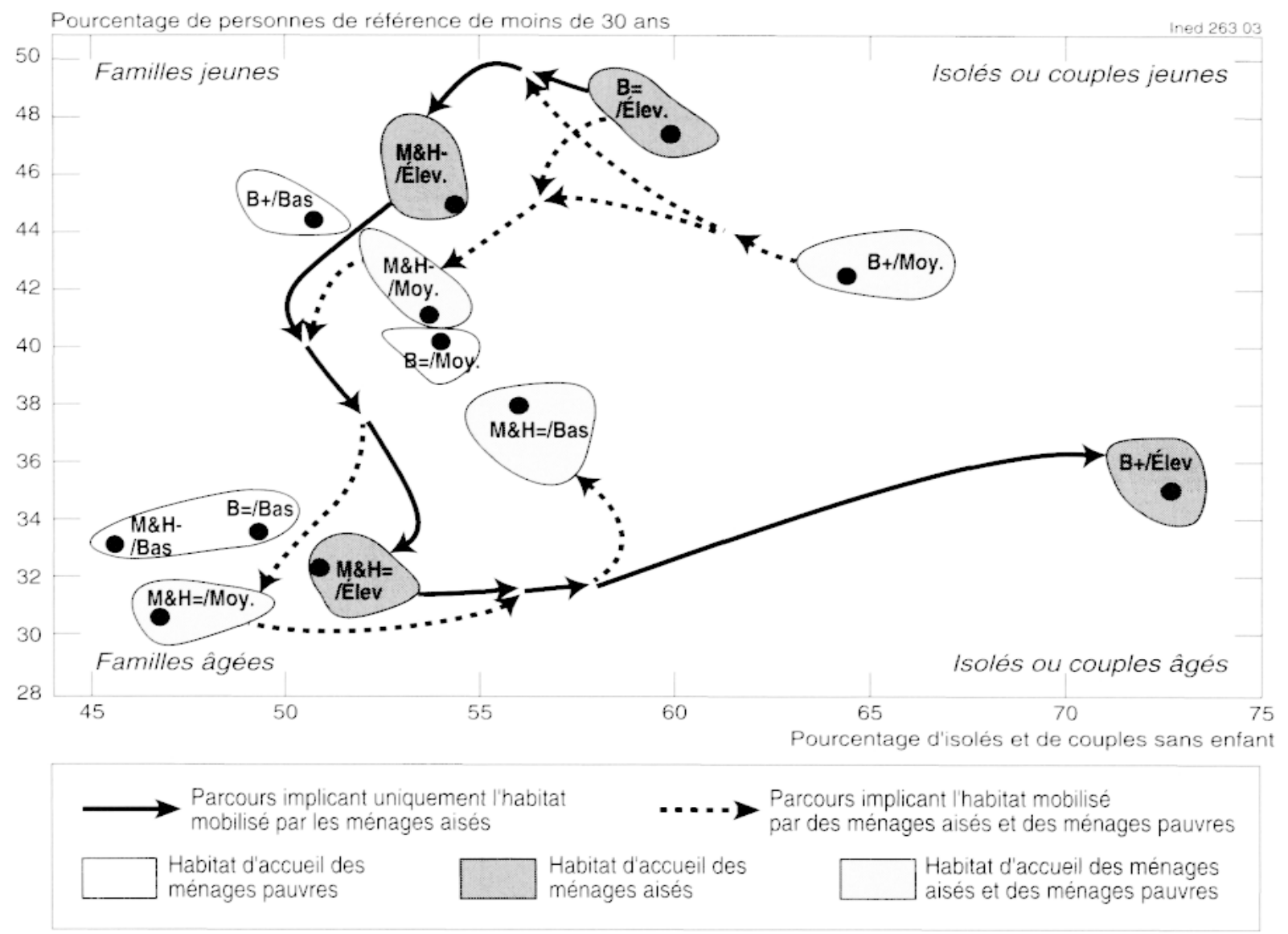

Figure 5. - Parcours résidentiels du moment des ménages aisés

logements spécialisés dans l'accueil des plus modestes (en blanc dans la figure 6). Dans ce cas, le parcours résidentiel des ménages les moins aisés s'effectue dans des zones de moins en moins valorisées : des secteurs bas de gamme qui s’embourgeoisent $(B+)$, soit vers ceux ancrés dans la paupérisation $(B=)$, soit vers des zones d’habitat haut de gamme qui se dévalorisent ( $\mathrm{M} \& \mathrm{H}-$ ). Les trajectoires sont alors descendantes.

Là encore. le passage par un habitat d'accueil de populations aux revenus diversifiés (trames pointillées dans la figure 6) crée des alternatives au parcours idéal typique descendant des ménages modestes. Une observation fine montre cependant que le passage par ces logements n inverse pas nécessairement le sens d une trajectoire descendante, soit en termes de statut du logement occupé, soit en termes de marquage social de la zone de résidence. Reste que ce type d’habitat offre aux ménages modestes en fin de parcours résidentiel des opportunités pour accéder aux secteurs les plus embourgeoisés du département ( $M \& H=$ ) et occuper les logements au statut socio-résidentiel bas ou moyen de ces zones.

Les analyses des parcours du moment mettent done en évidence une forte territorialisation des trajectoires résidentielles descendantes ou 


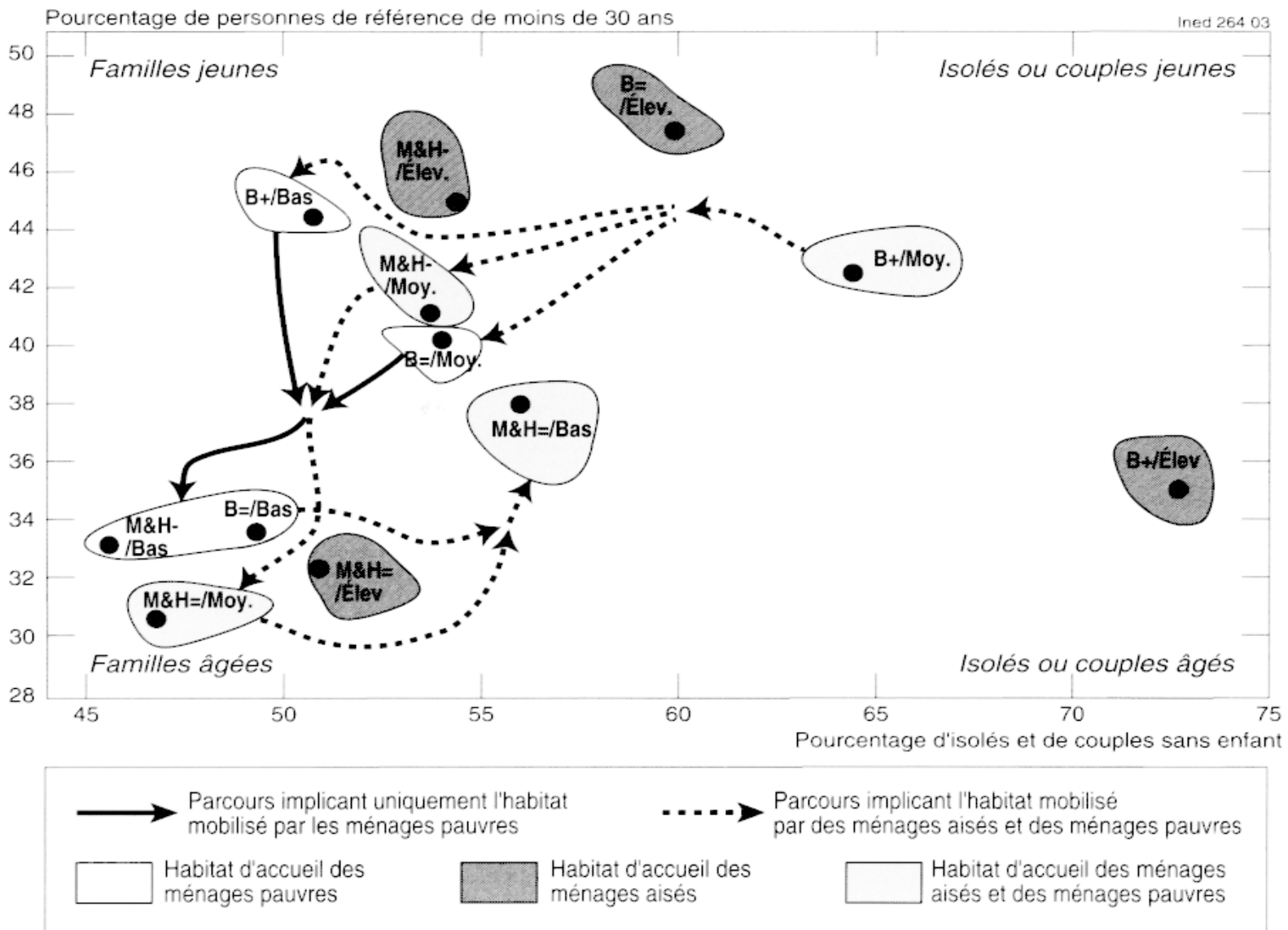

Figure 6.- Parcours résidentiels du moment des ménages aux faibles revenus

ascendantes réalisées dans le département. Les ménages circulant entre les communes du département opèrent leurs choix résidenticls en considérant à la fois le statut socio-résidentiel des logements et le marquage social des communes. En prenant en compte les ressources inégales des communes, ces arbitrages aboutissent à la constitution de filières de mobilité cloisonnées par les hiérarchies locales des statuts résidentiels des logements et par les mécanismes d'embourgeoisement et de dévalorisation sociale à l'œuvre. Ils participent à la production des marquages sociaux et des effets locaux et, de ce fait, à la transformation des spécialisations socioterritoriales pré-construites par les structures locales du parc immobilier.

\section{Conclusion}

Trois objectifs de méthode ont guidé cette recherche : relier les mouvements aux structures existantes, dans la mesure où les déplacements des ménages s'inscrivent toujours dans des habitats et des peuplements déjà en place; choisir ou construire des indicateurs aussi expressifs et synthé- 
tiques que possible, permettant de qualifier en même temps les mouvements et les structures; modéliser des interactions ou des dynamiques jugées importantes. Ces objectifs nous ont permis de comparer l'habitat et les mouvements résidentiels observés dans différentes communes d'un département confronté à une crise économique et sociale.

Les résultats ont montré que les effets d'âge et de cycle de vie, les structures sociales et les conjonctures locales pesaient fortement sur les dynamiques de sortie de crise. Le système résidentiel de la Seine-SaintDenis est producteur d'inégalités spatiales: les marquages sociaux des aires résidentielles sont fortement différenciés, et seules quelques communes possèdent les ressources locales nécessaires pour attirer des ménages extérieurs vecteurs d'une mixité sociale et d'un renouvellement du peuplement. Ce renouvellement repose en grande partie sur l'implantation dans des logements au statut moyen, les moins stables dans la hiérarchie résidentielle. Globalement cependant, les mouvements résidentiels internes à la Seine-Saint-Denis sont majoritaires : ils favorisent le maintien ou l'arrivée des ménages les plus fragilisés dans des logements au statut bas ou dans les secteurs géographiques les moins valorisés, et leur éviction des secteurs les plus valorisés. La majorité des habitants mobiles réalisent des trajectoires horizontales entre des communes au marquage social similaire et entre des classes d'habitat de même statut sociorésidentiel. Seule une minorité d'entre eux parvient à améliorer son statut résidentiel, soit en changeant de localisation, soit en changeant de classe d'habitat. Ces dynamiques mettent en évidence le rôle de la localisation à la fois dans les choix résidentiels et dans la détermination des trajectoires des ménages en mobilité.

Ces résultats conduisent à relativiser le rôle du statut d'occupation du logement dans la réalisation d'un parcours résidentiel ascendant dominant dans la société française. À l'opposé, ils conduisent à donner toute leur importance à la hiérarchie des statuts socio-résidentiels et aux effets territoriaux dans l'étude des dynamiques de peuplement des parcs immobiliers, dans celle des parcours résidentiels et du sens de leurs trajectoires. 


\section{ANNEXE}

Arborescence des 31 types de logements dans le département de la Seine-Saint-Denis en 1990

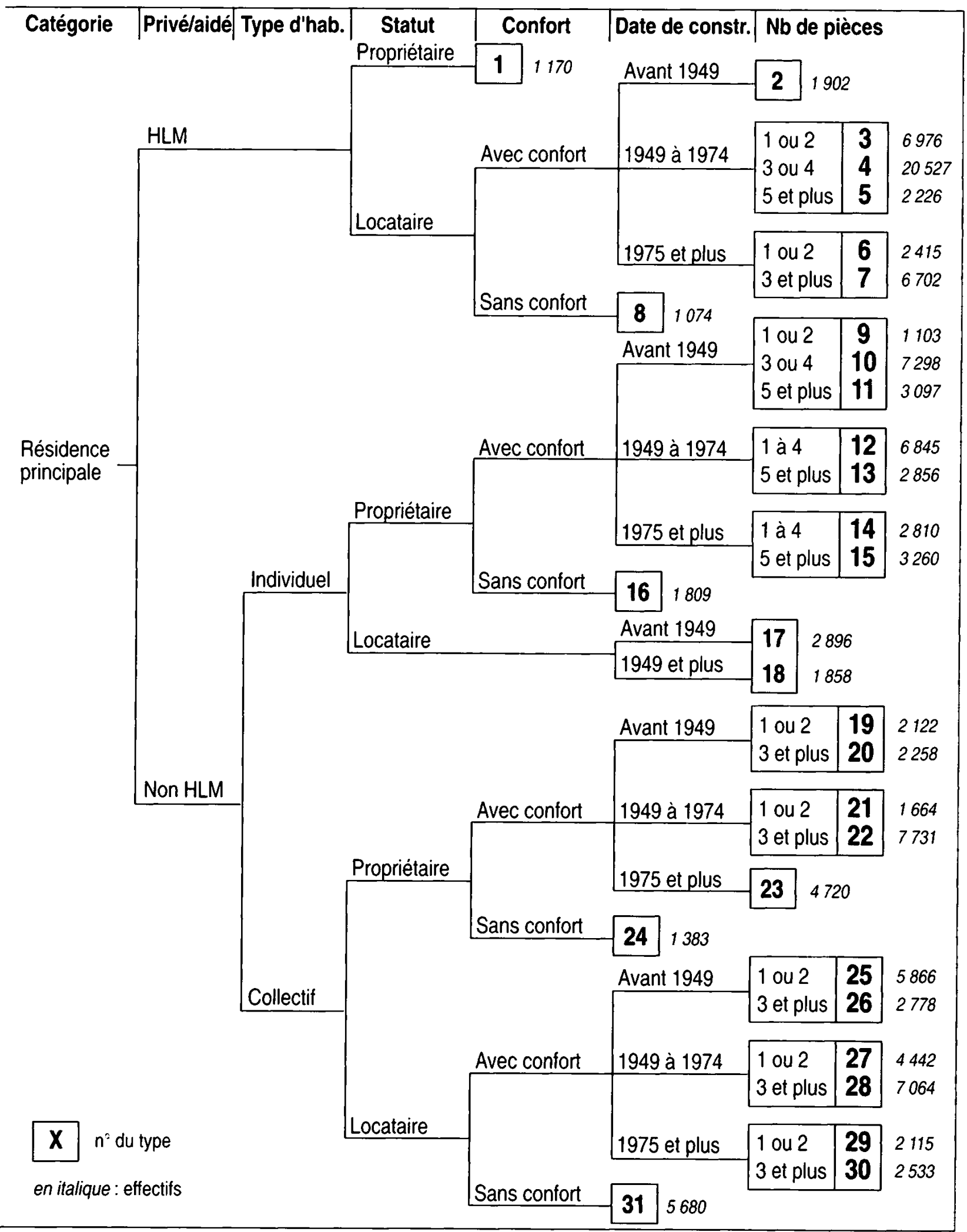

Source: Insee, recensement général de la population de 1990 (effectifs du sondage au quart). 


\section{RÉFÉRENCES}

ARBoNill.1: D. BoNvalet C.. 1992, “Conditions de logement et types de ménages. Résultats de l'enquête logement 1988 ». Population. 47(5), p. 1294-1306.

Baccaivi B.. 2000. "Projet de mobilité et cadre de vie ". in Mattei M.-F.. Pumain D.. Données urbaines. 3, Paris, Anthropos, p. 91-111.

BALIAIX R.. BIAIS J.-P. et al., 1984. Évolution du patrimoine logement et trajectoires résidentielles, Paris, PCA.

Blikgitr M.. RHeix C.. 1988. "Parc de logements, structures dés ménages et division sociale de l'espace en île-de-France ». Strates. 3.

BoNWAI:T C.. 1997. "Accession à la propriété et trajectoires individuelles". in Grafmeyer Y., Dansereau F. (éd.). Trajectoires familiales et espaces de vie én miliea urbain. L.yon. Presses universitaires de Lyon.

Brtx J.. RHI:I C. (éd.). 1994. la ségrégation dans la ville. Concepts et mesures, Paris. L. Harmattan.

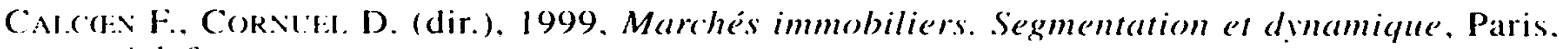
Adef.

Chalvit. L.. 1998. Le destin des générations, structure sociale et cohortes ent France au $X X^{\prime}$ sicele. Paris, Puf, $301 \mathrm{p}$.

CLARK W. A.V.. ONAKA J.-L.. 1983. "Life cycle and housing adjustment as explanation of residential mobility ". Urban Suludies. 20.

CIARK W. A.V., DIEI.MA.X F. M., 1996. Households and Housing. Choice and Outcomes in the Housing Market, New Jersey. Center for Urban and Policy Research.

ColRCAL: D., 1985. "Interaction between spatial mobility family and career life cycle: French survey ". European Sociological Revieu, 1(2).

Colrcital: D.. Plimaln D.. 1993. "Mobilité par temps de crise ", Population et Sociélés, 279.

DrMavibox A.. 1921. "L Labitation en France. Fssai de classification des principaux types". Annales de Ciéographie.

DeNaN O. D.. DuNCAN B., 1955, «A methodological analysis of segregation indexes », American Sociological Review. 20(2).

GAlbert P.. Iвво: S.. TITIN C.. 1996. « Segmented real estate markets and price mechanisms: The case of Paris ". International Jommal of Urban and Regional Research, 20(2).

LI: Bras H., Chesnals J.-C.. 1976, "Cycle de vie et âge des habitants ", Population, 31(2), p. 269-298.

L Ll:Il:vRE F́., 1992, "Mobilité résidentielle en France : observation et analyse. le point de vue du démographe ». in Lelièvre É.. Lévy-Vroelant C. (éd.). La ville en monvement: habitat ('t habitants. Paris, L'Harmattan. p. 21-30.

Li:VY J.-P.. 1995. "Les dynamiques socio-spatiales des marchés immobiliers". Relue d"économie régionale et urbaine. 3 .

Li:YY J.-P.. 1998a, "Les dynamiques du peuplement résidentiel ". Sociétés contemporaines, 29.

LíVY J.-P.. 1998b. "Habitat et habitants: position et mobilité dans lespace résidentiel". in Gratmeyer Y., Dansereau F. (éd.). Trajectoires familiales el espaces de vie én milieu urhain. Lyon. Presses universitaires de Lyon.

L.I:Y J.-P.. 2002, "The population make-up of residential areas: Structure and changes (Douai. France) . Journal of Housing and the Built Emironment. 17(3).

IJIYY J.-P.. HAl:MONT A.. 1997, L'offre de logements et la mobilité des ménages dans le départeme'nt de la Seine-Saint-Dénis, Nanterre. Centre de recherche sur l'habitat.

Livy J.-P.. Hat MoNi A., 2001. La mobilité résidemielle dans le Douatisis. Lille. Éditions de IORHA. 
LÉvy Jean-Pierre.- Peuplement et trajectoires dans I'espace résidentiel : le cas de la SeineSaint-Denis

L'article porte sur les processus de transformation du peuplement du département de la Seine-Saint-Denis (banlieue nord et est de Paris, 5000(0) ménages), du triple point de vue des logiques résidentielles des groupes sociaux, des effets de la structure de l'offre et des marquages sociaux des territoires.

À partir des données du recensement général de la population de 1990, on regroupe en classes d'habitat des types de logements dont les habitants ont des caractéristiques proches. Ces classes sont ensuite hiérarchisées et interprétées à partir d'un indice de statut sociorésidentiel, dont les variations spatiales nous permettent d'identifier l'influence des marquages sociaux des communes sur le peuplement (aires résidentielles), qu'ils relèvent des composantes de leur parc immobilier (zones d’habitat) ou de leur situation géographique (effets locaux ).

Ces typologies sont ensuite utilisées pour interpréter la mobilité résidentielle des ménages entrés dans un logement de la Seine-Saint-Denis en 1994. Les données relatives aux 1568 ménages enquêtés ont été exploitées dans une triple direction : pour cerner les trajectoires résidentielles des ménages; pour mettre à jour les choix de localisation des ménages, les marges de manceuvre et les contraintes pesant sur leur décision; en utilisant les parcours résidentiels du moment comme un indicateur des évolutions des peuplements territoriaux.

\section{Lévy Jean-Pierre.- Population Patterns and Household Trajectories in the Residential Milieu: the Example of the Seine-Saint-Denis}

This article examines the processes underlying changes in the residential population of the Seine-Saint-Denis department (a suburban district to the north and east of Paris, containing 500,000 households) from three points of view: 1) the residential logics of different social groups. 2) the effects of the housing supply structure and 3) of the social markers attributed to the different local areas.

Data from the 1990 Census are used to establish housing categories containing housing types whose residents have similar characteristics. These categories are then ranked and interpreted using an index of socio-residential status, whose spatial variations indicate the influence of the social markers of the communes on the population (residential areas), which can derive from the composition of their housing stock (housing zones) or from their geographical situation (local effects).

These typologies are then used to interpret the residential mobility of households who moved into a dwelling in the Seine-Saint-Denis in 1994. Data pertaining to the 1,568 surveyed households are analysed in three ways: to determine the residential trajectories of households: to shed light on the locational choices of households, and on the scope for action and constraints shaping their decision; and current residential histories are used as an indicator of changes in local populations.

\section{LAEY Jean-Picrre.- Población y trayectorias residenciales: el ejemplo de Sena- San Denis}

Este artículo analiza el proceso de transformación del poblamiento del departamento de Sena- San Denis (distrito del area metropolitana, situado al noreste de París, 500.000 hogares) desde tres perspectivas: las estrategias residenciales de diferentes grupos sociales, el impacto de la estructura de la oferta y las demarcaciones o estructura social del territorio.

A partir de los datos del censo general de población de 1990 se construyen categorías de habitat en base a tipos de vivienda cuyos residentes tienen características similares. Estas categorías son ordenadas jerárquicamente y se interpretan en base a un índice de status socioresidencial, las variaciones espaciales del cual permiten estudiar la influencia de la categoría social del municipio sobre su poblamiento (areas residenciales), tanto en lo relativo al parque inmobiliario (zonas de vivienda) como a su situación geográfica (efectos de localización).

Estas tipologías sirven de base para estudiar la movilidad residencial de los hogares que se instalaron en Sena - San Denis en 1994. La explotación de los datos relativos a los 1,568 hogares encuestados tiene tres objetivos: observar las trayectorias residenciales de los hogares, describir la selección de localización de los hogares, las opciones disponibles y las restricciones existentes a la hora de tomar una decisión. Las trayectorias residenciales se utilizan como indicador de la evolución del poblamiento territorial.

Jean-Pierre LF́VY, CNRS/CRH-Louest (UMR 7145 du CNRS), courriel : jean-pierre.levy $@$ paris-valdescine.archi.fr 